\title{
Extracellular vesicle release and uptake by the liver under normo- and hyperlipidemia
}

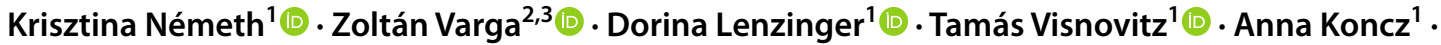

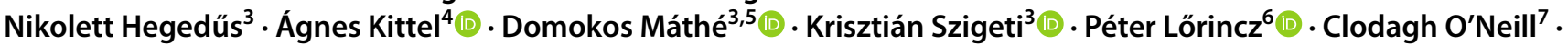

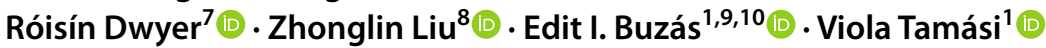

Received: 11 June 2021 / Revised: 3 September 2021 / Accepted: 5 October 2021 / Published online: 19 October 2021

(c) The Author(s) 2021

\begin{abstract}
Liver plays a central role in elimination of circulating extracellular vesicles (EVs), and it also significantly contributes to EV release. However, the involvement of the different liver cell populations remains unknown. Here, we investigated EV uptake and release both in normolipemia and hyperlipidemia. C57BL/6 mice were kept on high fat diet for 20-30 weeks before circulating EV profiles were determined. In addition, control mice were intravenously injected with ${ }^{99 \mathrm{~m}} \mathrm{Tc}$-HYNICDuramycin labeled EVs, and an hour later, biodistribution was analyzed by SPECT/CT. In vitro, isolated liver cell types were tested for EV release and uptake with/without prior fatty acid treatment. We detected an elevated circulating EV number after the high fat diet. To clarify the differential involvement of liver cell types, we carried out in vitro experiments. We found an increased release of EVs by primary hepatocytes at concentrations of fatty acids comparable to what is characteristic for hyperlipidemia. When investigating EV biodistribution with ${ }^{99 \mathrm{~m}} \mathrm{Tc}$-labeled EVs, we detected EV accumulation primarily in the liver upon intravenous injection of mice with medium $(326.3 \pm 19.8 \mathrm{~nm})$ and small EVs $(130.5 \pm 5.8 \mathrm{~nm})$. In vitro, we found that medium and small EVs were preferentially taken up by Kupffer cells, and liver sinusoidal endothelial cells, respectively. Finally, we demonstrated that in hyperlipidemia, there was a decreased EV uptake both by Kupffer cells and liver sinusoidal endothelial cells. Our data suggest that hyperlipidema increases the release and reduces the uptake of EVs by liver cells. We also provide evidence for a size-dependent differential EV uptake by the different cell types of the liver. The EV radiolabeling protocol using ${ }^{99 \mathrm{~m}} \mathrm{Tc}$-Duramycin may provide a fast and simple labeling approach for SPECT/CT imaging of EVs biodistribution.
\end{abstract}

Keywords Extracellular vesicle $\cdot$ Extracellular particles $\cdot$ Hepatocyte $\cdot$ Kupffer cell $\cdot$ Liver sinusoidal endothelial cells

Edit I. Buzás and Viola Tamási shares last authorship.

Viola Tamási

viola.tamasi@gmail.com

1 Department of Genetics, Cell- and Immunobiology, Semmelweis University, Budapest, Hungary

2 Research Centre for Natural Sciences, Institute of Materials and Environmental Chemistry, Budapest, Hungary

3 Department of Biophysics and Radiation Biology, Semmelweis University, Budapest, Hungary

4 Eötvös Loránd Research Network, Institute of Experimental Medicine, Budapest, Hungary

5 Hungarian Centre of Excellence for Molecular Medicine, In Vivo Imaging ACF, Budapest, Hungary

$\begin{array}{ll}\text { Abbreviations } \\ \text { BSA } & \text { Bovine serum albumin } \\ \text { EV } & \text { Extracellular vesicle } \\ \text { FBS } & \text { Fetal bovine serum }\end{array}$

6 Department of Anatomy, Cell and Developmental Biology, Eötvös Loránd University, Budapest, Hungary

7 Discipline of Surgery, National University of Ireland, Lambe Institute for Translational Research, Galway, Ireland

8 Department of Medical Imaging, University of Arizona, Tucson, AZ, USA

9 Hungarian Centre of Excellence for Molecular Medicine, Semmelweis University Extracellular Vesicle Research Group, Budapest, Hungary

10 ELKH-SE Immune-Proteogenomics Research Group, Budapest, Hungary 


$\begin{array}{ll}\text { FFA } & \text { Free fatty acid } \\ \text { GFP } & \text { Green fluorescent protein } \\ \text { HDL-C } & \text { High-density lipoprotein cholesterol } \\ \text { HEP } & \text { Hepatocyte } \\ \text { HFD } & \text { High fat diet } \\ \text { KC } & \text { Kupffer cell } \\ \text { LDL-C } & \text { Low-density lipoprotein cholesterol } \\ \text { lEV } & \text { Large-sized extracellular vesicle } \\ \text { LSEC } & \text { Liver sinusoidal endothelial cell } \\ \text { mEV } & \text { Medium-sized extracellular vesicle } \\ \text { NPC } & \text { Non-parenchymal cell } \\ \text { NTA } & \text { Nanoparticle tracking analysis } \\ \text { OA } & \text { Oleic acid } \\ \text { PA } & \text { Palmitic acid } \\ \text { PBS } & \text { Phosphate buffer saline } \\ \text { PFA } & \text { Paraformaldehyde } \\ \text { POt } & \text { Palmitic acid/oleic acid treatment } \\ \text { RT-qPCR } & \text { Real-time quantitative polymerase chain } \\ & \text { reaction } \\ \text { SD } & \text { Standard deviation } \\ \text { SEC } & \text { Size exclusion chromatography } \\ \text { sEV } & \text { Small-sized extracellular vesicle } \\ \text { TEM } & \text { Transmission electron microscope } \\ \text { TFF } & \text { Tangential flow filtration } \\ \text { VLDL-C } & \text { Very low-density lipoprotein cholesterol } \\ & \end{array}$

\section{Introduction}

Extracellular vesicles (EV) are mostly spherical, phospholipid bilayer-enclosed particles [1]. They were originally thought to be carriers for the removal of cellular waste [2]. However, we now know that due to their complex molecular composition (including nucleic acids, lipids, proteins and carbohydrates), they are significant players of cell-cell communication [3, 4]. The composition of EVs is determined by the types and functional states of the donor cells. The versatile roles of EVs have been confirmed in several physiological and pathological processes [5]. Even though the classification of EVs is based on their biogenesis, often it is not possible to determine the biogenetic origin of EVs. Sizebased subfractions of EVs include large EVs (lEVs, $>1 \mu \mathrm{m}$ ), medium EVs (mEVs, $150 \mathrm{~nm}$ to $1 \mu \mathrm{m}$ ) and small EVs (sEVs $\leq 100 \mathrm{~nm})[6]$.

Biodistribution studies of EVs have shown that they primarily accumulate in liver and spleen [7-13]. According to Imai and co-workers, even after depletion of hepatic macrophages (Kupffer cells, KCs), EVs accumulated in the liver [12]. This suggests that liver cell populations other than KCs are also involved in EV removal from the circulation. Both parenchymal hepatocytes (HEPs) and non-parenchymal cells (NPCs) are capable of endocytosis suggesting a potential strong EV scavenger function liver cells in the body [14].
Although the rapid clearance of EVs by liver has already been demonstrated [7-12], the relative contribution of the individual liver cell types to EV release and elimination has not been investigated yet.

Currently, over 3 million adults (2020) have been diagnosed with primary or secondary hyperlipidemia in Europe and in the United States, with the number of cases increasing dramatically [15]. Cardiovascular diseases often associated with hyperlipidemia represent the number one cause of death worldwide [16]. The high-fat Western diet is known to increase the likelihood of developing secondary, dietinduced hyperlipidemia. We were compelled to carry out this study, because we hypothesized that the level of circulating lipoprotein particles may have an impact on $\mathrm{EV}$ release and uptake by the liver. Of note, higher blood lipid levels may lead to lipotoxicity, which, in turn, may also alter the dynamics of EV production by liver cells [17-19].

Here, we report that hyperlipidemia indeed has a major impact on the dynamics of hepatic EV production and uptake. These results may have significant implications for the development of EV-based therapies.

\section{Materials and methods}

\section{Animal experiments}

All mouse experiments followed the European Union's Council Directive (86/609/EEC), and were approved by the Semmelweis University's Institutional Animal Care and Use Committee (PE/EA/1655-7/2018). Male, C57BL/6 mice were housed in a temperature-controlled $\left(25^{\circ} \mathrm{C}\right)$ room, with normal light cycle (12-12 h), and were given free access to food and water. At the age of 6 weeks, mice were divided into two groups and were fed either by a high fat diet (HFD, $45 \mathrm{kcal} \%$ fat, D12451, Research Diets, USA) or by a normal mouse diet for up to 20 and 30 weeks (HFD-20 and HFD30 , respectively). Throughout the experimental period, the body weight was measured two-weekly. At the end of the high fat/normal diet, mice were fasted for five hours prior to glucose tolerance test. The glucose levels were measured using a Dcont Ideal instrument (Dcont, Hungary) from blood obtained by cutting off the end of the tail before and 30 and $90 \mathrm{~min}$ after the intraperitoneal injections of glucose (20 $\mathrm{w} / \mathrm{v} \%, 2 \mathrm{~g} / \mathrm{kg}$ ). Plasma levels of low-density lipoprotein cholesterol (LDL-C)/ very low-density lipoprotein cholesterol (VLDL-C) and high-density lipoprotein cholesterol (HDLC) were determined with a HDL and LDL/VLDL Quantification Kit (Sigma, USA). Hepatic triglyceride levels were measured using a Triglyceride Colometric Assay Kit (Cayman Chemical, USA). For total RNA, 40-50 mg pieces of the liver were homogenized with a Micropestle (Geneaid, Taiwan) in QIAzol Lysis Reagent (QIAGEN, Germany), 
and total RNA was isolated with a Blood/Cell Total RNA Mini Kit (Geneaid, Taiwan). cDNA was synthesized using SensiFAST cDNA Synthesis Kit (Bioline, UK), from $1 \mu \mathrm{g}$ of total RNA. Real-time quantitative PCR (RT-qPCR) was performed using a 7900 HT Fast Real-Time PCR System (Applied Biosystems, USA), using SensiFAST Probe HiROX Kit (Bioline, UK) according to the instruction of the manufacturer. Briefly, $50 \mathrm{ng}$ of cDNA in a final volume of $10 \mu \mathrm{L}$ was amplified using $0.5 \mu \mathrm{L}$ of Taqman assay and 5 $\mu \mathrm{L}$ of SensiFAST Probe Hi-ROX mix. Taqman assays were used for Gapdh (Mm9999915_g1), Cd36 (Mm00432403_ m1), Olr1 (Mm00454586_m1), Ldlr (Mm01177349_m1), Scarb1 (Mm00450234_m1), Cyp3a11 (Mm00731567_m1), Cyp4a14 (Mm00484132_m1), Pparg (Mm01184322_m1), Ppara (Mm00440934_m1), Lxr (Mm00437265_g1), Car (Mm01283980_g1), Hnf4a (Mm01247712_m1) (ThermoFisher, USA). The fold changes of mRNA were determined using $2^{-\Delta \Delta \mathrm{Ct}}$ method, using Gapdh as an internal control.

\section{Separation of murine blood plasma EVs}

EVs were separated from plasma of control (normal diet) and HFD mice. The blood samples were collected from the inferior vena cava in tubes containing Acid Citrate Dextrose solution A (ACD A, Greiner Bio-One, Hungary), after performing euthanasia of the mice with $\mathrm{CO}_{2}$. The ACD tubes were used to prevent in vitro vesiculation [20]. The blood samples were spun once at $1500 \times g$ for $15 \mathrm{~min}$ [21]. Based on the protein and particle content, protein/particle ratio, $\mathrm{EV}$ and non-EV markers, we found that the number of centrifugations in plasma isolation protocol did not affect the EV content (Supplementary Fig. 1). Plasma samples were snap frozen and stored at $-80{ }^{\circ} \mathrm{C}$. EVs were separated by differential centrifugation combined with size exclusion chromatography (SEC, qEV Original/70 nm, IZON, NewZealand). The thawn up samples were diluted twofold with $0.2 \mu \mathrm{m}$ filtered phosphate buffer saline (PBS). The samples were centrifuged at $2000 \times g, 4^{\circ} \mathrm{C}$ for $30 \mathrm{~min}$. The $\mathrm{mEV}$ fraction was separated at $12,500 \times g, 4{ }^{\circ} \mathrm{C}$ for $40 \mathrm{~min}$. The supernatant was centrifuged at $100,000 \times g, 4{ }^{\circ} \mathrm{C}$ for $70 \mathrm{~min}$ to pellet sEVs. EV-containing pellets were resuspended in $500 \mu \mathrm{L} 0.2 \mu \mathrm{m}$ filtered PBS and were further purified with SEC. After rinsing the column with PBS, samples were applied onto the column and were fractionated according to the manufacturer's protocol: a pooled EV fraction of 1500 $\mu \mathrm{L}$ was collected and the presence of EVs was confirmed both by flow cytometry and nanoparticle tracking analysis (NTA, ZetaView, ParticleMetrix GmbH, Germany). EVs were aliquoted, snap frozen and stored at $-80{ }^{\circ} \mathrm{C}$ until use. The detailed parameters of the centrifugation steps are summarized in Supplementary Table 2. The presence of EVs in the preparation was confirmed by transmission electron microscopy (TEM, Supplementary Fig. 2).

\section{Isolation of primary liver cells}

Mice were anesthetized with a mixture of ketamine-xylazine (12-1.6 mg/mL, Medicus Partner Kft., Hungary). Liver cells were isolated by anterograde two-step collagenase perfusion according to F. Cabral et al., 2018, with minor modifications [22]. Briefly, pre-warmed $\left(42^{\circ} \mathrm{C}\right.$, Precision GP05 Wather Bath, ThermoFisher, USA), oxygen-enriched (VisionAire5 oxygen concentrator, AirSep Corporation, USA) solutions were used at a flow rate of $4 \mathrm{~mL} / \mathrm{min}$ (EP-1 Econo pump, BioRad, USA). Desmosomes were disrupted with solution A (PBS + 0.5 M EGTA). Subsequently, the connective tissue of the liver was digested with solution $B[3.9 \mathrm{~g} / \mathrm{L} \mathrm{NaCl}$, $0.5 \mathrm{~g} / \mathrm{L} \mathrm{KCl}, 0.7 \mathrm{~g} / \mathrm{L} \mathrm{CaCl}_{2}, 50 \mathrm{mM}$ HEPES (ThermoFisher, USA) and collagenase IV ( $0.3 \mathrm{mg} / \mathrm{mL}$, Worthington, USA $)]$. After the perfusion and subsequent excision of the liver, it was disrupted mechanically, and the tissue remnants were removed with a $100 \mu \mathrm{m}$ filter (Miltenyi Biotec, Germany).

\section{Hepatocyte monoculture}

Separated liver cells were processed as follows. HEPs were pelleted by low-speed centrifugation $\left(50 \mathrm{~g}, 3 \mathrm{~min}, 4^{\circ} \mathrm{C}\right)$, the viable cells were isolated with a $40 \%$ Percoll solution (Sigma, USA, $150 \mathrm{~g}, 7 \mathrm{~min}, 4{ }^{\circ} \mathrm{C}$ ). The cells were cultured in Type I collagen-coated plates $\left(15 \mu \mathrm{g} / \mathrm{cm}^{2}\right.$, Sigma, USA) and were seeded in either 24-well plates for EV uptake studies $\left(1.5 \times 10^{5}\right.$ cells/well $)$ or in 6 -well plates for EV production studies $\left(10 \times 10^{5}\right.$ cells/well $)$ in a seeding medium (DMEM (high glucose, $4.5 \mathrm{~g} / \mathrm{L}$ ), $10 \%$ fetal bovine serum (FBS, Gibco), $1000 \mathrm{U} / \mathrm{L}$ penicillin, $1000 \mu \mathrm{g} / \mathrm{L}$ streptomycin, $2 \mathrm{mmol} / \mathrm{L}$ L-glutamine, $7.5 \mathrm{mg} / \mathrm{L}$ Hydrocortisone, $0.02 \mathrm{mg} / \mathrm{L}$ epidermal growth factor, $0.014 \mathrm{mg} / \mathrm{L}$ glucagon, $1 \mathrm{mM}$ sodium pyruvate, $0.5 \mathrm{ml} / \mathrm{L}$ insulin (all from Sigma, USA)). The cells were allowed to attach to the plate surface for $2-3 \mathrm{~h}$ at $37{ }^{\circ} \mathrm{C}$ and $5 \% \mathrm{CO}_{2}$. After the incubation, the medium was changed to a FBS-free seeding medium.

\section{Fatty acid treatment}

We simulated hyperlipidemic conditions in vitro by exposing cells to free fatty acids (FFA) for $16 \mathrm{~h}$. Oleic acid (OA) and palmitic acid (PA) were dissolved in DMEM (high glucose, $4.5 \mathrm{~g} / \mathrm{L}$ ) containing $10 \%$ bovine serum albumin (BSA, Sigma, USA) with overnight rotation (10 rpm, Fisherbrand $^{\mathrm{TM}}$ MiniTube Rotator, ThermoFisher, USA). Stock solutions of FFA were further diluted in FBS-free seeding medium to a final concentration of $400 \mu \mathrm{M} \mathrm{OA}$ and $200 \mu \mathrm{M}$ PA. 


\section{Assessment of the production of albumin and uric acid}

Albumin production of HEPs was measured using a Mouse Albumin ELISA Kit (Abcam, UK). Uric acid concentration in the conditioned medium was analyzed using Amplex ${ }^{\mathrm{TM}}$ Red Uric Acid/Uricase Assay Kit (ThermoFisher, USA).

\section{Separation of hepatocyte-derived EVs}

EVs were separated from serum-free conditioned medium of HEPs collected $16 \mathrm{~h}$ after HEPs isolation from $5 \times 10^{6}$ control and $5 \times 10^{6}$ FFA-treated cells. The protocol used for plasma EV separation was followed for hepatocyte-derived EV separation with minor modifications. Briefly, before the $2000 \times g$ centrifugation step, the conditioned medium was centrifuged at $300 \times g, 20{ }^{\circ} \mathrm{C}$ for $10 \mathrm{~min}$ and filtered $(5 \mu \mathrm{m}$, Millipore, USA) to remove cells. The detailed parameters of the centrifugation steps are summarized in Supplementary Table 3. The presence of EVs was confirmed by TEM (Supplementary Fig. 3).

\section{Separation of HEK293T-palmGFP-derived EVs}

HEK293T-palmGFP cell line (generous gift of Xandra Breakefield and Charles Lai) was grown in DMEM (low glucose, $1 \mathrm{~g} / \mathrm{L})$ medium, supplemented with $10 \%$ FBS (Gibco, USA), $1000 \mathrm{U} / \mathrm{L}$ penicillin, $1000 \mu \mathrm{g} / \mathrm{L}$ streptomycin, $2 \mathrm{mmol} / \mathrm{L} \mathrm{L-glutamine} \mathrm{(Sigma,} \mathrm{USA).} \mathrm{Cells} \mathrm{were}$ maintained at $37{ }^{\circ} \mathrm{C}$ in a humidified atmosphere containing $5 \% \mathrm{CO}_{2}$. Conditioned medium $(160 \mathrm{~mL})$ was collected $24 \mathrm{~h}$ after culture in serum-free medium in $8 \mathrm{~T}-175 \mathrm{TC}$ treated flasks (Eppendorf, Germany). The passage number of cells ranged from 8 to 18 , reaching $70-80 \%$ confluence, representing $131.7 \pm 42.8 \times 10^{6}$ cells per separation, with a viability of $90.1 \pm 2.0 \%$. Flow cytometric analysis of apoptosis in HEK293T-palmGFP cells is shown in Supplementary Fig. 4. The ratio of total apoptotic cells was below $7 \%$. Cell cultures were regularly monitored for Mycoplasma. The conditioned medium was centrifuged at $300 \times g, 20{ }^{\circ} \mathrm{C}$ for $10 \mathrm{~min}$ and filtered ( $5 \mu \mathrm{m}$, Millipore, USA) to remove cells. The supernatant was centrifuged at $2000 \times g, 4{ }^{\circ} \mathrm{C}$ for $30 \mathrm{~min}$ to pellet $\mathrm{lEVs}$. The mEVs were then separated at $12,500 \times g, 4{ }^{\circ} \mathrm{C}$ for $40 \mathrm{~min}$. The supernatant was concentrated and further purified using tangential flow filtration (TFF-Easy, $20 \mathrm{~nm}$ pore size, HansaBioMed Estonia) and was centrifuged at $100,000 \times g, 4{ }^{\circ} \mathrm{C}$ for $70 \mathrm{~min}$ to pellet sEVs. After separation, the EV fractions were re-suspended in $0.2 \mu \mathrm{m}$ filtered PBS, aliquoted, were snap frozen and were stored at $-80{ }^{\circ} \mathrm{C}$ until use. The detailed parameters of the centrifugation steps are summarized in Supplementary Table 4.

\section{Hepatocyte: non-parenchymal liver cell (NPC) co-culture}

Hepatocytes were isolated as described above. Additional liver cell populations were isolated as published earlier, with minor modifications [23, 24]. Briefly, isolated HEPs were allowed to attach to a 24 -well plate for $1 \mathrm{~h}$ at $37{ }^{\circ} \mathrm{C}, 5 \% \mathrm{CO}_{2}$, then collagen Type I was overlaid on the cells $\left(15 \mu \mathrm{g} / \mathrm{cm}^{2}\right.$, Sigma, USA). The collagen was allowed to form a gel for $45 \mathrm{~min}$, while NPCs were isolated from the HEP supernatant by differential centrifugation $\left(300 \times g, 10 \mathrm{~min}, 4{ }^{\circ} \mathrm{C}\right)$. The viable cells were isolated with $33 \%$ Percoll solution $(700 \times g$, $12 \mathrm{~min}, 4^{\circ} \mathrm{C}$ ) and live HEPs were removed by centrifugation (1. $50 \times g, 3 \mathrm{~min}, 4{ }^{\circ} \mathrm{C} ; 2.70 \times g, 3 \mathrm{~min}, 4{ }^{\circ} \mathrm{C}$ ). NPCs were seeded on HEPs at a density of $1 \times 10^{5}$ cells/well in FBS-free seeding medium. After $2 \mathrm{~h}$ of incubation, the medium was changed and non-adherent cells were removed.

\section{Oil red 0 staining}

Triglyceride accumulation of liver cells was visualized by Oil Red O (Sigma, USA) staining. Cells were fixed with $4 \%$ paraformaldehyde (PFA) in PBS, washed with PBS and stained with $60 \%$ Oil Red O diluted with distilled water. After washing with PBS and distilled water, cells were examined with a Nikon Diaphot TMD Inverted Microscope (Nikon, Japan).

\section{Immunocytochemistry}

Cells were grown on coverslips (12 mm, VWR, USA) for $16 \mathrm{~h}$ after isolation, were washed with PBS and fixed with $4 \%$ PFA. Then, samples were blocked with PBS containing 5\% FBS, supplemented with $0.3 \%$ Triton X-100 (Molar Chemicals Kft., Hungary) for intracellular albumin labeling. HEPs (albumin positive), KCs (F4/80 positive), and LSECs (CD146 positive) were identified in a co-culture by antibodies to cell-specific markers by immunocytochemistry. Primary antibodies were incubated overnight at $4{ }^{\circ} \mathrm{C}$ with the indicated dilutions (rabbit polyclonal anti-human serum albumin, 1:100; mouse monoclonal anti-CD146, 1:100; rat monoclonal anti-F4/80-eF660, 1:100). After washing out the primary antibodies, the secondary antibodies were incubated for $2 \mathrm{~h}$ at $37^{\circ} \mathrm{C}$ with shaking (goat polyclonal anti-rabbit IgG-AF700, 1:100; goat polyclonal anti-mouse IgG-eF570, 1:100). Samples were washed with PBS, distilled water and covered with ProLongTM Gold antifade reagent with DAPI (ThermoFisher, USA). The slides were examined with Leica TCS SP8 Confocal Laser Scanning Microscope (Leica, Germany). All antibodies used in these experiments are listed in Supplementary Table 1. 


\section{Cytokine secretion measurements}

Cytokine production of liver cells was measured from the conditioned medium using a LEGENDplex ${ }^{\mathrm{TM}}$ Mouse Macrophage/Microglia Panel 13-plex (BioLegend, USA) and CytoFLEX S flow cytometer (Beckman Coulter, USA) according to the instruction of the manufacturer.

\section{Transmission electron microscopy of EVs}

The morphology of EVs was examined by TEM. Samples were prepared according to Théry et al. 2006 with minor modifications [25]. Briefly, $2 \mu \mathrm{L}$ of a sample was placed onto the surface of a formvar-coated grid, and was incubated for $10 \mathrm{~min}$ at RT. The residual solution was removed and fixed with $2 \%$ glutaraldehyde for $10 \mathrm{~min}$. The grids were washed three times for $5 \mathrm{~min}$, and we used uranyl oxalate for $10 \mathrm{~min}$ for contrast enhancement. The samples were further contrasted and embedded in a mixture of $4 \%$ uranyl acetate and $2 \%$ methyl cellulose and were examined by JEOL 1011 transmission electron microscope (Japan). HEK293TpalmGFP-derived $\mathrm{mEVs}$ were also visualized using a different approach. Transmission electron microscopy of ultrathin sections of EV pellets was carried out (Supplementary Fig. 5). In this case, the mEVs of the $12.5 \mathrm{k}$ pellet were processed as described previously [26].

\section{Nanoparticle tracking analysis (NTA) of EVs}

The size distribution of EVs was analyzed by NTA. The measurements were performed on a ZetaView PMX-120 instrument using ZetaVIEW software. The detailed parameters of the measurements are summarized in Supplementary Table 5.

\section{Protein and lipid assays of EVs}

The protein concentration was measured by Micro BCA assay (ThermoFisher, USA). The lipid content was assessed by the optimized sulfo-phospho-vanillin (SPV) lipid assay according to Visnovitz et al., 2019 [27]. The lipid content of EVs was used as a basis of normalization in our EV uptake studies.

\section{Flow cytometry of EVs}

EV-associated markers were analyzed by flow cytometry (CytoFLEX S, Beckman Coulter, USA). Both sEVs and mEVs secreted by HEK293T-palmGFP cells were bound onto the surface of latex beads $(3 \mu \mathrm{m}, 4 \% \mathrm{w} / \mathrm{v}$ Aldehyde/ Sulfate Latex Beads, ThermoFisher, USA) overnight at $4{ }^{\circ} \mathrm{C}$. One $\mu \mathrm{g}$ lipid/sample sEVs $\left(\sim 8 \times 10^{9}\right.$ particles $)$ and mEVs $\left(\sim 4 \times 10^{8}\right.$ particles $)$ were bound onto the beads. In the case of plasma and HEP-derived EVs, identical volumes $(200 \mu \mathrm{L})$ of the $1500 \mu \mathrm{L}$ pooled SEC fraction were co-incubated with the beads. Afterwards, the beads were blocked with $100 \mathrm{mM}$ glycine and $2.5 \%$ w/v BSA solution. HEK293T-palmGFP EVs were stained with AxV-AF647 (1:100), mouse antihuman monoclonal CD63-PerCP (1:100), mouse anti-human monoclonal CD81-PerCP/Cy5.5 (1:100), mouse anti-human monoclonal CD9-APC (1:100), mouse anti-human monoclonal ApoB (1:100) labeled with goat anti-mouse polyclonal IgG-eF570 (as detailed in Supplementary Table 1).

Plasma/HEP EVs were stained with AxV-Pacific Blue (1:100), rat anti-mouse monoclonal CD63-APC (1:200), hamster anti-mouse monoclonal CD81-PE (1:400), mouse anti-human monoclonal ApoB (1:100) labeled with goat anti-mouse polyclonal IgG-eF570 (as detailed in Supplementary Table 1). Five thousand events/sample were measured by flow cytometry. Data were analyzed using FlowJo (Becton Dickinson, USA). In addition to the \% positivity of the beads for the selected markers, the relative mean fluorescence intensity (RMFI) was also determined, which may provide additional information on the number of particles associated with a single bead [28].

\section{Flow cytometry analysis of EV uptake}

In the in vitro experiments, liver cells were detached from the plate surface with Accutase solution (Sigma, USA) and were analyzed directly after a centrifugation step $(50 \times g$, 3 min at $4{ }^{\circ} \mathrm{C}$ ). NPC cells were separated from HEPs by centrifugation. The pellet was re-suspended in a special buffer to provide optimal conditions for liver cell survival and subsequent immunostaining (1:20 dilution of MACS BSA Stock Solution with autoMACS Rinsing Solution, Miltenyi Biotec, Germany) containing FcR Blocking Reagent (1:10). After 10 min incubation, the excess reagent was removed by centrifugation, and the cells were re-suspended in solution (rat anti-mouse monoclonal CD146-PerCP-Vio700, 1:50; recombinant human anti-mouse monoclonal F4/80-PE, 1:100 in MACS buffer). After washing out the antibodies, the cells were stained with TO-PRO-3 (1:3000) and measured by flow cytometry. Within the population of living cells, CD146 ${ }^{+}$ (LSECs) and $\mathrm{CD} 146^{-}$cells were gated. $\mathrm{F} 4 / 80^{+}(\mathrm{KCs})$ cells were then identified within the $\mathrm{CD} 146^{-}$population according to Lynch et al., 2018 (Supplementary Fig. 6) [29]. All antibodies and viability dye used in these experiments are listed in Supplementary Table 1.

\section{Detection of EV fluorescence by a fluorescence-labeled organism bioimaging instrument}

The GFP positivity of the HEK293T-palmGFP-derived EVs was demonstrated with Fluorescence-labeled Organism 
Bioimaging Instrument (FOBI, Neoscience, Suwon City, Rep. Korea) under certain parameters ("Blue channel" light source $(455 \mathrm{~nm})$ and $520 \mathrm{~nm}$ long pass emission filter for detection). The exposure time was $1000 \mathrm{~ms} / \mathrm{image}$ with gain level 1. After sample preparation, the fluorescence of the HEK293T-palmGFP-derived sEVs $(1 \mu \mathrm{g}$ lipid in $28 \mu \mathrm{L})$, mEVs ( $1 \mu \mathrm{g}$ lipid in $12 \mu \mathrm{L})$, and PBS as control sample were measured in Eppendorf tubes. Prior to every fluorescence scan, background image was registered in the same position using white light illumination $(400-800 \mathrm{~nm})$ without applying any filter for detection. In these cases, the exposure time was $100 \mathrm{~ms}$ with gain level 1 . For image analysis, fluorescence images were co-registered to background scans by VivoQuant software (inviCRO, Boston, US).

\section{Radiolabeling of HEK293T-palmGFP-derived EVs and in vivo SPECT/CT imaging}

Radiolabeling of HEK293T-palmGFP-derived sEV and $\mathrm{mEVs}$ was performed with the ${ }^{99 \mathrm{~m}} \mathrm{Tc}-\mathrm{HYNIC}$-Duramycin kit (Molecular Targeting Technologies, Inc, U.S.A.). In the first step, HYNIC-Duramycin was labeled with ${ }^{99 \mathrm{~m}} \mathrm{Tc}$ according to the manufacturer's instructions. $3.5 \mathrm{GBq}\left[{ }^{99 \mathrm{~m}} \mathrm{TcO} 4\right]^{-}$was eluted in $0.5 \mathrm{~mL}$ saline and added to the kit, followed by placing the vial at $80{ }^{\circ} \mathrm{C}$ for $20 \mathrm{~min}$. Radiochemical yield of this step was checked with reversed-phase HPLC [30], and found to be greater than $95 \%$. Next, $900 \mathrm{MBq}{ }^{99 \mathrm{~m}} \mathrm{Tc}-$ HYNIC-Duramycin in $200 \mu \mathrm{L}$ was added to $60 \mu \mathrm{L}$ sEV and $\mathrm{mEV}$ samples ( $3 \mu \mathrm{g}$ total lipid in each) and diluted with 240 $\mu \mathrm{L}$ PBS and incubated at $30^{\circ} \mathrm{C}$ for 20 min with shaking at $200 \mathrm{rpm}$. Purification of the labeled EVs was performed by SEC using a $V_{\mathrm{T}}=3.5 \mathrm{~mL}$ column filled with Sepharose CL-2B gel (GE Healthcare Bio-Sciences AB, Sweden). $0.5 \mathrm{~mL}$ sample was pipetted on the column, and $0.5 \mathrm{~mL}$ fractions were collected using PBS as eluent. The fraction corresponding to the void volume of the column (from 1 to $1.5 \mathrm{~mL}$ elution volume) contained the radiolabelled EVs. Radiochemcial purity was checked by radio-HPLC-SEC using a Tricorn 5/100 glass columns (GE Healthcare BioSciences AB, Sweden), filled with Sepharose CL-2B [31].

In vivo imaging was performed with a NanoSPECT/CT Silver Upgrade (Mediso Ltd, Hungary) sequential animal SPECT/CT imaging system. In the SPECT/CT experiment, $12 \pm 3 \mathrm{MBq}$ of ${ }^{99 \mathrm{~m}} \mathrm{Tc}-\mathrm{HYNIC}-$ Duramycin labeled sEV and $\mathrm{mEV}$ samples in $100 \mu \mathrm{L}$ volume was injected into the tail vein of C57BL/ 6 mice ( $n=2$ for both $\mathrm{sEV}$ and $\mathrm{mEV}$ samples). During the scans, the animals were continuously anaesthetized using a mixture of $1-1.5 \%$ isoflurane and medical oxygen, and their body temperature was maintained at $37{ }^{\circ} \mathrm{C}$ throughout the scanning. $\mathrm{CT}$ and subsequent SPECT imaging started $1 \mathrm{~h}$ after the time of administration, and the acquisition lasted 10.5 and $30 \mathrm{~min}$, respectively. Reconstructed images were analyzed with Fusion (Mediso
Ltd, Hungary) and VivoQuant (inviCRO LLC, US) image analysis software by placing appropriate Volume of Interests (VOI) manually on the organs in each CT scans. Radioactivity concentrations in $\mathrm{MBq} / \mathrm{cm}^{3}$ were determined for each VOI and corrected for scattering and isotopic decay in the reconstruction algorithm. The uptake values were measured in the heart, lungs, kidneys, bladder, liver, spleen and bones.

\section{Statistical analysis and graphic software}

Values are reported as mean \pm standard deviation (SD). Statistical analyses were performed using GraphPad Prism 7.00 (USA). The normal distribution of the data was examined using the Shapiro-Wilk normality test. For normally distributed data, unpaired/paired $T$ test or one-way/two-way ANOVA test was performed. Post hoc analyses of significance were made by Tukey's multiple comparisons test. If the data did not pass the normality test, a Mann-Whitney test was performed. $p<0.05$ was accepted as statistically significant. The figures were generated using GraphPad Prism 7.00 (USA).

\section{Results}

\section{Characterisctics of HFD-fed mice}

HFD increases the risk to develop metabolic disorder [32]. Here, we found a significant increase in the body weight even after 1 week on HFD (Fig. 1a, f). The mean body weight increased by $17.7 \pm 9.2 \%$ and $26.2 \pm 5.9 \%$ in the HFD-20 and HFD-30 groups, respectively. Continued weight gain was observed at 20 and 30 weeks (by $72.8 \pm 8.9 \%$ and $42.0 \pm 15.8 \%$, respectively). The HFD also affected blood sugar levels (Fig. 1b, g). The blood glucose levels in the HFD groups were significantly higher after $6 \mathrm{~h}$ fasting than in the control groups. Following intraperitoneal glucose injection, blood glucose levels in the HFD groups remained elevated during the time of the experiment $(90 \mathrm{~min})$. To determine dyslipidemia, plasma LDL-C/VLDL-C and HDL-C levels were measured (Fig. 1c, h). Compared to the control groups, the HFD significantly increased plasma LDL-C/VLDL-C and HDL-C levels. In hyperlipidemia, liver accumulates triglycerides (TG) that can lead to fatty liver (Fig. 1d, i). Liver TG levels were significantly higher in both the HFD-20 and the HFD-30 groups. The expression of genes important in the pathogenesis of fatty liver was examined by RT-qPCR (Fig. 1e, j). The gene expression of many receptors involved in the uptake of lipoproteins and FFAs were altered. In the HFD-20 group, there was a significant increase in the $C d 36, O \operatorname{Orl}$ and a decrease in $L d l r$ and Scarbl expression. Furthermore, the mRNA levels 

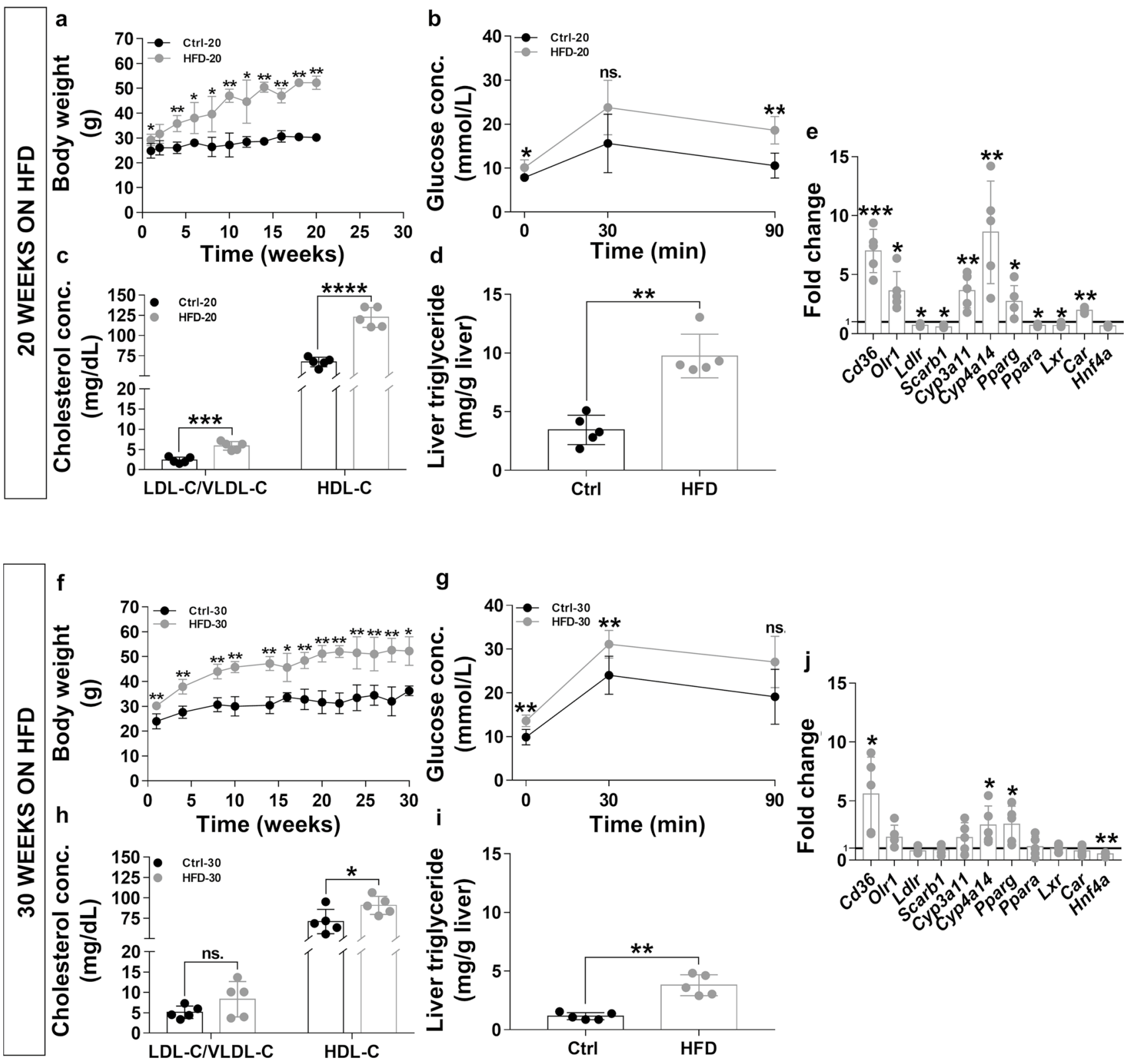

Fig. 1 Effect of HFD on body weight, glucose tolerance, plasma HDL-C and LDL-C/VLDL-C levels, liver TG concentration and mRNA expression levels. The effect of HFD on body weight was monitored regularly (a and f). After 20 and 30 weeks of HFD, the fasting glucose levels and glucose tolerance were investigated (b and g). The development of hyperlipidemia was confirmed by the HDL-C and LDL-C/VLDL-C measurements (c and h). We examined the

of Cyp3al1 and Cyp4al4 enzymes associated with fatty liver development were increased. The nuclear receptors, implicated in the metabolism and storage of FFAs showed the following pattern: Pparg, Car mRNA levels were increased, while Ppara and $L x r$ were decreased. In the HFD-30 group, only the Cd36, Cyp4al4 and Pparg expression was upregulated. In contrast, the expression of Hnf $4 a$ was significantly reduced. accumulation of hepatic TG in response to hyperlipidemia (d and i). We also investigated changes in liver mRNA expression using RTqPCR (e and $\mathbf{j}$ ). Values are reported as mean \pm SD. $p$ values are calculated by Mann-Whitney test (a and $\mathbf{f})$ or unpaired $T$ test $(\mathbf{b}, \mathbf{c}, \mathbf{d}$, $\mathbf{e}, \mathbf{g}, \mathbf{h}, \mathbf{i}$ and $\mathbf{j}) . n=5$ was in each group. $n s$ not-significant, $*<0.05$, $* *<0.01, * * *<0.001, * * * *<0.0001$

\section{Effect of hyperlipidemia on the plasma EV content}

Next, we investigated the effect of HFD on the concentration of circulating plasma EVs. Based on the analysis of $\mathrm{EV}$ markers, there was no change in the plasma mEV content (Fig. 2a, Supplementary Fig. 7a). ApoB ${ }^{+}$, mEV-coated beads were detected at a strikingly higher frequency and higher RMFI value as compared to $\mathrm{AxV}, \mathrm{CD} 63$ and $\mathrm{CD} 81$ 

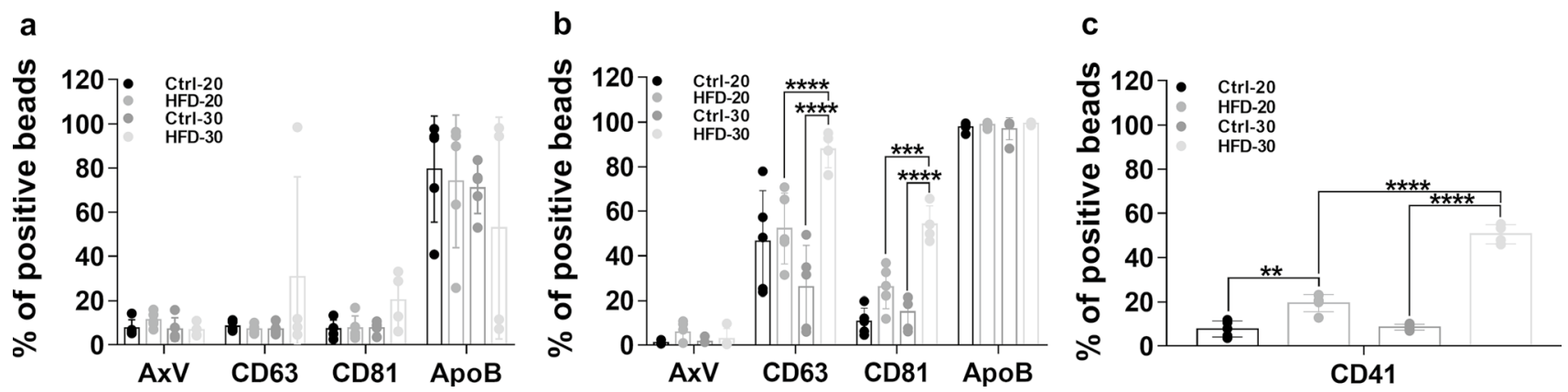

Fig. 2 Flow cytometric characterization of plasma-derived EVs based on their EV-specific marker expression. The effect of HFD on plasma EV content was determined by EV marker expression. EVs were purified by SEC and $200 \mu \mathrm{L}$ of the pooled $1500 \mu \mathrm{L}$ EV-containing elutes were bound onto latex beads. HFD did not affect the plasma $\mathrm{mEV}$ content (a). Bead-bound plasma sEVs resulted in a significantly increased CD63, CD81 and CD41 positive bead frequency in

positive ones. The staining pattern of bead-bound sEVs was different from what we have seen in the case of $\mathrm{mEV}$. While $\mathrm{AxV}^{+}$beads were present at low frequency, we found high frequency for CD63, CD81 and ApoB (Fig. 2b) sEVs compared to mEVs. The proportions of $\mathrm{CD} 63^{+}$and $\mathrm{CD} 81^{+} \mathrm{sEVs}$ were significantly increased in the HFD-30 group (based on the number of the positively stained beads). However, there was no significant difference in CD63, CD81 RMFI values (Supplementary Fig. 7b). ApoB positivity was tested, because $A p o B$ is known to co-isolate with EVs [33]. Interestingly, $\mathrm{ApoB}^{+}$particles/ApoB molecules were present on $100 \%$ of the beads, regardless of group (Fig. 2b). In contrast, the RMFI value of ApoB was significantly higher in the HFD-20 and HFD-30 groups (Supplementary Fig. 7b), which correlates well with plasma LDL-C/VLDL-C concentration. We tested $\mathrm{CD} 41^{+} \mathrm{EVs}$, because it is known that $\mathrm{CD} 41^{+}$platelet-derived EVs can induce platelet aggregation, and thus, may contribute to the development of cardiovascular diseases [34, 35]. It can be clearly seen that HFD significantly increases the percentage of CD41 $1^{+}$beads after 20 and 30 weeks (Fig. 2c). Additionally, the RMFI value of CD41 was significantly higher in the HFD-30 group (Supplementary Fig. 7c). The results of the antibody control experiments are shown in the Supplementary Table 6. We were also interested if there was any difference in the size distribution of EVs in the blood plasma of mice kept of HFD for 20 or 30 weeks. Based on our NTA measurements, we found no difference in the size distribution of EVs. The median size (X50), particle concentration and protein/particle number ratio of the EVs are shown in the Supplementary Table 7.

\section{Characteristics of liver cell cultures}

Primary liver cells were cultured either in HEP monoculture or in HEP-NPC co-culture. Hyperlipidemic conditions the HFD-30 group as compared to the Ctrl-30 and HFD-20 groups (b and $\mathbf{c}$ ). Values are reported as mean $\pm \mathrm{SD}$. $p$ values were calculated by two-way ANOVA (a and b) or one-way ANOVA (c) and Tukey's multiple comparisons post hoc test. $n_{\mathrm{Ctrl}-20}=5, n_{\mathrm{HFD}-20}=5, n_{\mathrm{Ctrl}-30}=5$, $n_{\mathrm{HFD}-30}=4$ (one plasma sample was excluded due to significant hemolysis), $* *<0.01, * * *<0.001, * * * *<0.0001$

were in vitro simulated with the mixture of oleic $(400 \mu \mathrm{M})$ and palmitic $(200 \mu \mathrm{M})$ acids. As shown in Fig. 3a (left panel), cuboidal HEPs were grown in regular FBS-free seeding medium. They contained accumulated lipid droplets in their cytoplasms as identified by Oil Red O staining. Of note, the size of the lipid droplets in HEPs increased markedly after $16 \mathrm{~h}$ FFA treatment (Supplementary Fig. 8), while NPC cells apparently did not accumulate fat droplets (Fig. 3b, black arrows). It is known that in vitro conditions may lead to de-differentiation of HEPs leading to a decrease in specific cell functions [36]. Albumin production is considered as an important indicator of HEP function. The secreted albumin remained unchanged in control and palmitic acid/oleic acid treated (POt) culture during the course of the experiments (Fig. 3c). Uric acid production correlated positively with hyperlipidemia (as a marker of hepatic steatosis). It can be clearly seen that after $16 \mathrm{~h}$ FFA treatment, there is a significant increase in the uric acid production by HEPs (Fig. 3d). The liver cell co-culture contained HEP:LSEC:KC:other cells in 6:2:1:1 ratios (which is the case for normal mouse liver). HEPs $\left(1.5 \times 10^{5}\right)$ were cultured with $1 \times 10^{5}$ NPCs. The ratio of KCs to LSECs was validated by flow cytometry and was found $22.9 \pm 4.8 \%: 39.9 \pm 4.8 \%$ (Supplementary Fig. 9). The presence of HEPs (identified by intracellular albumin immunostaining), KCs (detected by F4/80 positivity), and LSECs (recognized by CD146 expression) in the coculture was confirmed (Fig. 3e, f, g). Different liver cell types were reported to secrete various cytokines [37, 38]. In line with this, testing our liver cell cultures, we also found evidence for the secretion of numerous cytokines (Supplementary Fig. 10). This cytokine environment was maintained even under hyperlipidemic conditions, where only the production of CXCL1 in HEP monoculture and the production of CCL22, GCSF, CXCL1 in HEP-NPC 
a

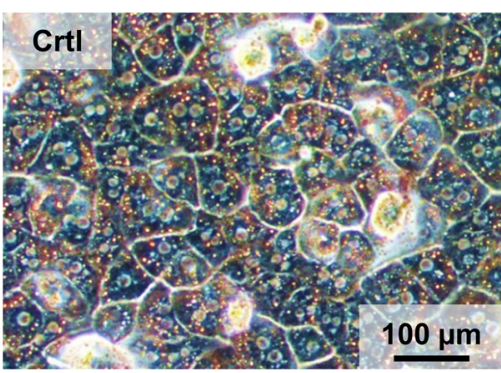

b

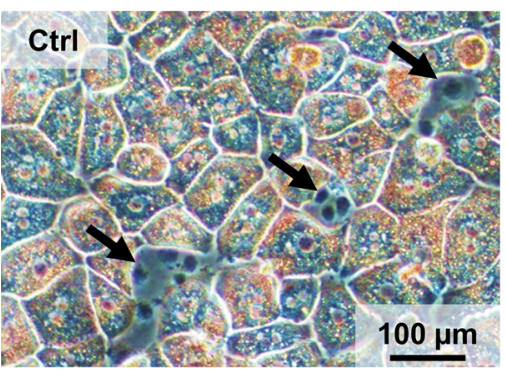

c

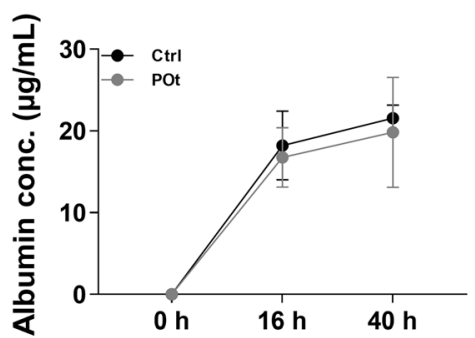

h

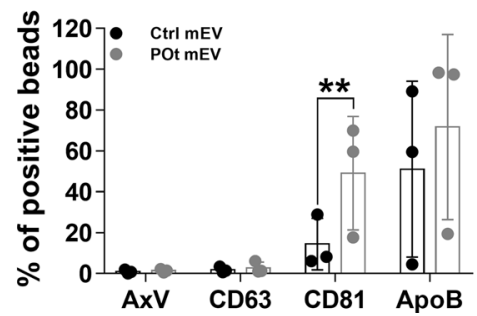

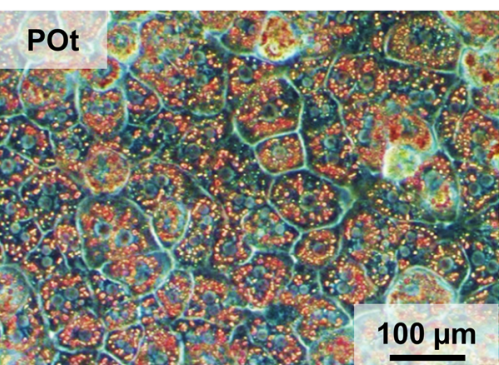

$100 \mu \mathrm{m}$

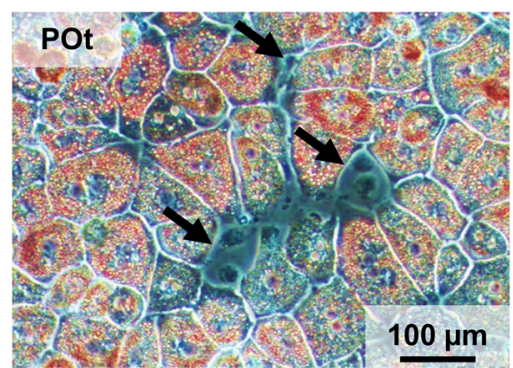

d
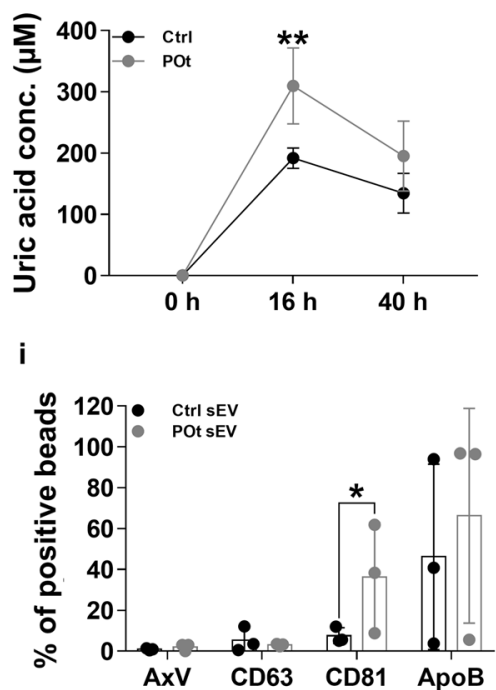
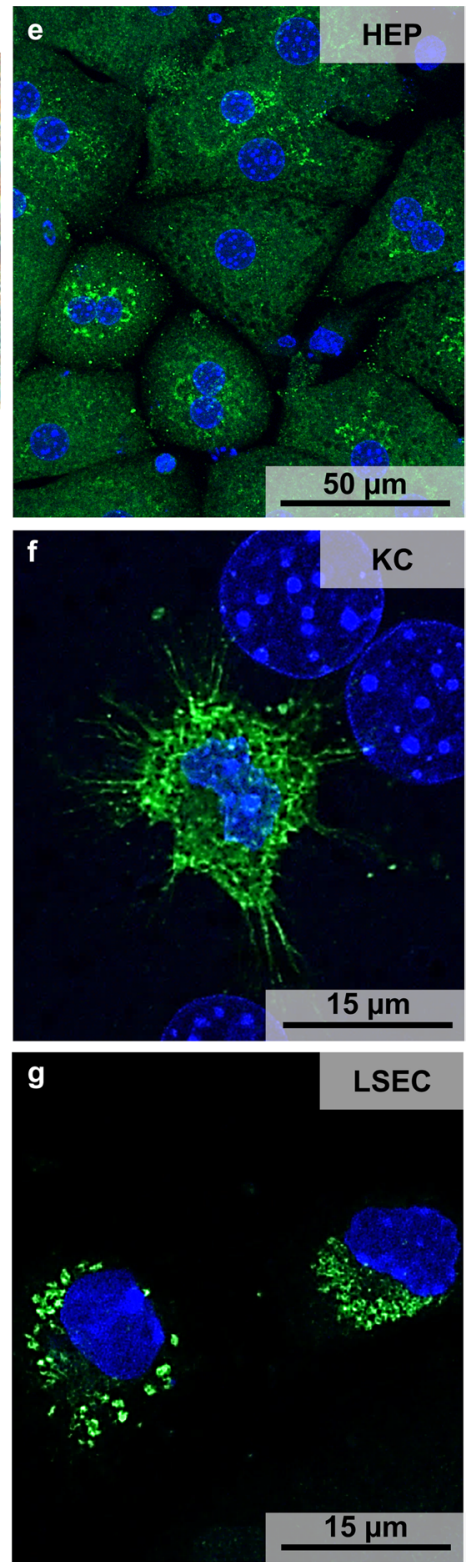

Fig. 3 Characterization of liver cell cultures and hepatocyte-derived EVs. In vitro, hyperlipidemic conditions were modeled by FFA treatment. Triglyceride accumulation in HEP monoculture (a) and HEPNPC co-culture (b) was demonstrated by Oil Red O staining. While no lipid accumulation was seen in NPCs (black arrows), there was a high number of lipid droplets in HEPs. HEPs rapidly de-differentiate in vitro, leading to decreased albumin production. During our experiments, albumin production remained unchanged (c). The production of uric acid as a marker of hyperlipidemia was significantly increased after FFA treatment (d). HEPs were identified in HEP-NPC co-cul- ture by their albumin production (e), KCs by F4/80 immunostaining (f), and LSECs by their CD146 cell surface expression (g). Low power magnification microphotographs are shown in Supplementary Fig. 12. The effect of POt on HEP-derived EVs was determined by EV marker expression. EVs were purified by SEC and $200 \mu \mathrm{L}$ of the $1500 \mu \mathrm{L}$ EV-containing elutes were bound to latex beads. HEPderived $\mathrm{CD}^{+} 1^{+} \mathrm{mEVs}$ and sEVs carrying bead frequencies were elevated in the POt group (h and $\mathbf{i}$ ). Values are reported as mean \pm SD. $\mathrm{p}$ values were calculated by two-way ANOVA and Tukey's multiple comparisons post-hoc test. $n_{\mathrm{Ctrl}}=3, n_{\mathrm{POt}}=3, *<0.05, * *<0.01$ co-culture were significantly increased. However, we did not detect a pronounced pro-inflammatory cytokine response.

\section{Effect of hyperlipidemic conditions on the release of hepatocyte-derived EVs}

Next, we studied the effect of hyperlipidemia on the EV production by HEPs in vitro. EVs were separated from serum free conditioned medium of control and POt cells. The 
proportion of both $\mathrm{AxV}^{+}$and $\mathrm{CD} 63^{+}$HEP-derived mEVs and SEVs were low, and was unaffected by FFA treatment. In contrast, POt significantly increased the proportion of both CD81 $1^{+} \mathrm{mEVs}$ (Fig. 3h) and sEVs (Fig. 3i). There was no statistically significant change in RMFI values of CD81 (Supplementary Fig. $7 d$ and e). The results of the antibody control experiments are shown in the Supplementary Table 6. Similarly to what we have found in the case of circulating EVs, our NTA measurements did not reveal any difference in the size and distribution of EVs released by HEPs under control and POt conditions. The median size (X50), particle concentration and protein/particle number ratio of the EVs are shown in the Supplementary Table 8.

\section{Characterization of HEK293T-palmGFP-derived EVs}

mEVs and sEVs were separated from the conditioned medium of HEK293T-palmGFP cell line and were characterized by TEM, NTA, protein and lipid content. The presence and characteristic morphology of EVs were demonstrated by TEM (Fig. 4a, b). Based on the NTA measurements (Fig. $4 \mathrm{c}$ and d), the median size (X50) of mEVs and sEVs were $326.3 \pm 19.8$ and $130.5 \pm 5.8 \mathrm{~nm}$, respectively. The protein/lipid ratio was found to be $2.2 \pm 1.1$ and $11.4 \pm 3.9$ for mEVs and sEVs, respectively (Fig. 4e). EV-associated markers were also identified using flow cytometry (Fig. 4f). We found a very prominent CD81 positivity of latex bead bound $\mathrm{mEV}$ s and sEVs. The results of the antibody control experiments are shown in Supplementary Fig. 11. The GFP fluorescence of $\mathrm{EV}$ fractions was shown by flow cytometry (Fig. 4g) and FOBI (Fig. 4h).

\section{In vivo biodistribution of HEK293T-palmGFP-derived EVs}

In our ${ }^{99 \mathrm{~m}} \mathrm{Tc}$-labeling experiments, we obtained a label yield of 60 to $80 \mathrm{MBq} / 3 \mu \mathrm{g}$ total lipid in $0.5 \mathrm{~mL}$ volume. As revealed by radio-HPLC-SEC, the purity of labeled EV samples was above 95\%, which is sufficient for in vivo investigations. Figure 5a, b shows typical SPECT/CT images of the in vivo biodistribution of sEV and mEVs. For both samples, high accumulation of the injected EVs can be observed in liver and spleen in accordance with previous literature data [39]. Radioactivity in the bladder, which does not succeed $3 \%$ of the total injected activity, can be attributed to free ${ }^{99 \mathrm{~m}} \mathrm{Tc}-\mathrm{HYNIC}$-Duramycin. This observation also indicates an in vivo stability of the labeling, because free ${ }^{99 \mathrm{~m}} \mathrm{Tc}-\mathrm{HYNIC}$-Duramycin is quickly eliminated through the kidneys with a low uptake in the liver and intestines (22 ID\%/g of the injected radioactivity can be detected in urine at $60 \mathrm{~min}$ after injection according to Zhao et al. [30]). Figure 5c shows the distribution of the labeled EVs within the different organs as a percentage of the whole body radioactivity. Liver uptakes of sEVs and $\mathrm{mEVs}$ were $69.5 \pm 2.5 \%$ and $69.7 \pm 0.7 \%$, respectively. Spleen showed the second largest accumulation of radioactivity with $4.8 \pm 1.0 \%$ and $5.5 \pm 1.0 \%$, for $\mathrm{mEVs}$ and $\mathrm{sEVs}$, respectively. In summary, in vivo SPECT/CT imaging with ${ }^{99 \mathrm{~m}} \mathrm{Tc}-\mathrm{HYNIC}$-Duramycin revealed that the liver takes up the majority of intravenously injected EVs, and no significant difference can be observed in this regard between sEVs and mEVs.

\section{EV uptake in hepatocyte monoculture}

HEPs comprise about $80 \%$ of the total cells and volume of the liver [40]. The question arises as to what extent they are able to internalize and eliminate EVs. To answer this question, we investigated the uptake of HEK293T-palmGFP EVs in HEP monoculture. In preliminary experiments, we found that EVs were taken up by HEPs in a dose dependent manner, reaching a maximum at $24 \mathrm{~h}$ (data not shown). Figure $6 \mathrm{a}, \mathrm{b}$ shows results obtained after incubation of $1 \mu \mathrm{g}$ lipid containing EVs with HEPs for $24 \mathrm{~h}$ in serum free condition. As demonstrated in Fig. 6a, b, HEPs were able to take up both mEVs and sEVs. Hyperlipidemic conditions did not affect the uptake of $\mathrm{mEVs}$ or sEVs significantly.

\section{EV uptake in HEP-NPC co-culture}

LSECs and KCs play a complementary role in elimination of macromolecules and particulates from blood circulation. To get a more precise picture of the role of the liver in the elimination of different nanoparticles, we examined the uptake of EVs in a HEP-NPC co-culture. EV uptake kinetics of HEPs, KCs and LSECs was examined in vitro (Fig. 6c, d). One $\mu$ g lipid containing HEK293T-palmGFP mEVs or SEVs were added to liver cells and were incubated for 2, 6 and $24 \mathrm{~h}$. After $6 \mathrm{~h}$, we saw an increased EV uptake, which reached a maximum at $24 \mathrm{~h}$ (Fig. 6c, d). Interestingly, while mEVs were taken up primarily by KCs (Fig. 6e), the sEVs were internalized predominantly by LSECs (Fig. 6f). Under hyperlipidemic conditions, accumulation of lipid droplets in liver cells increased. To study EV uptake under hyperlipidemic conditions, cells were pre-treated with a mixture of palmitic acid-oleic acids. After $16 \mathrm{~h}$ of FFA pre-treatment, liver cells were incubated with mEVs or sEVs for $24 \mathrm{~h}$. Our preliminary data showed that $\mathrm{EV}$ exposure shorter than $24 \mathrm{~h}$ did not affect the elimination of EVs. In contrast, we found under our in vitro hyperlipidemic model conditions significantly reduced $\mathrm{mEV}$ uptake by KCs and sEV by LSECs after $24 \mathrm{~h} \mathrm{EV}$ exposure. 


\section{a}

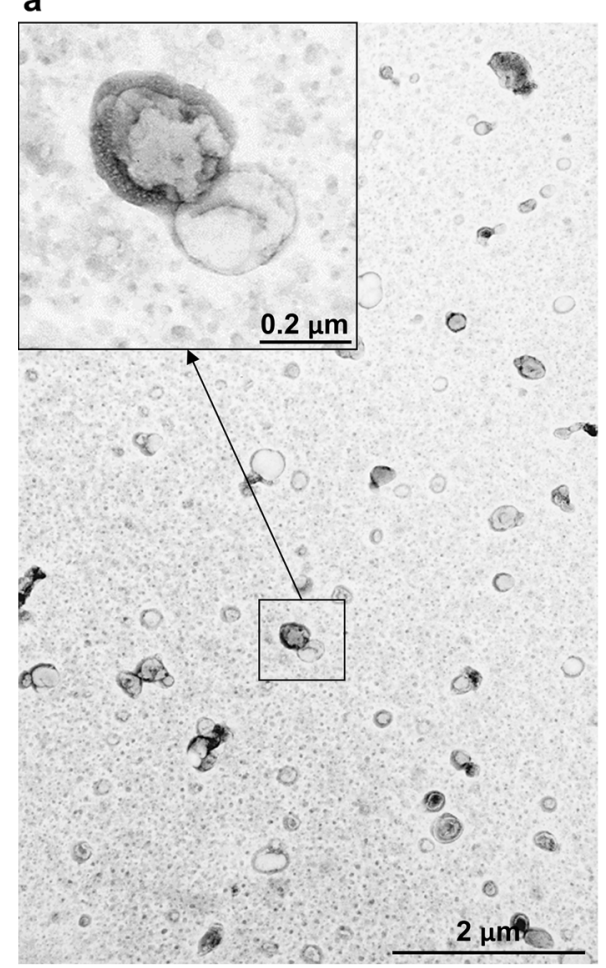

b

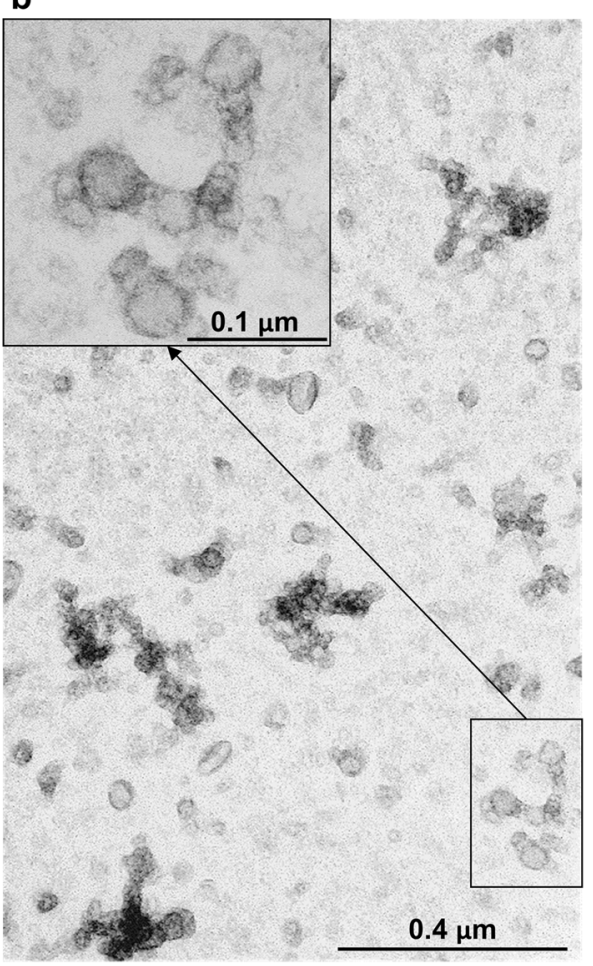

C

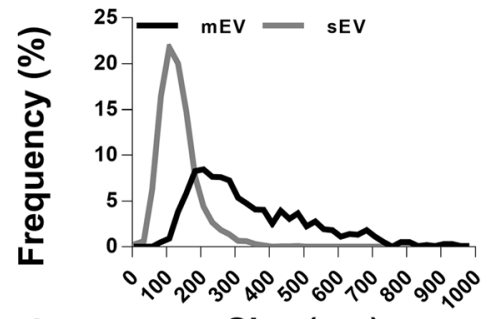

d
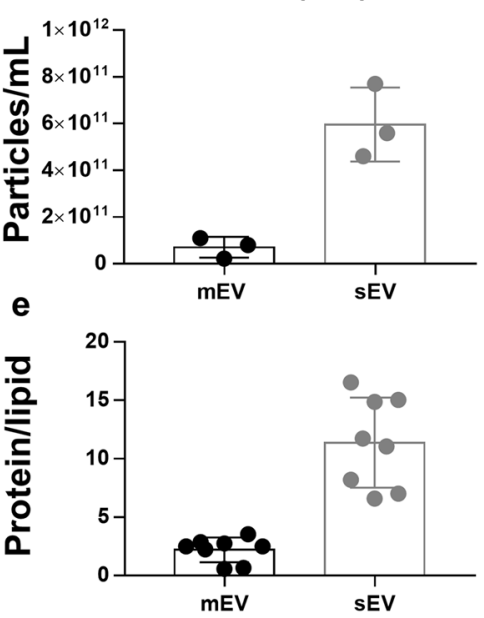

CD9

ApoB
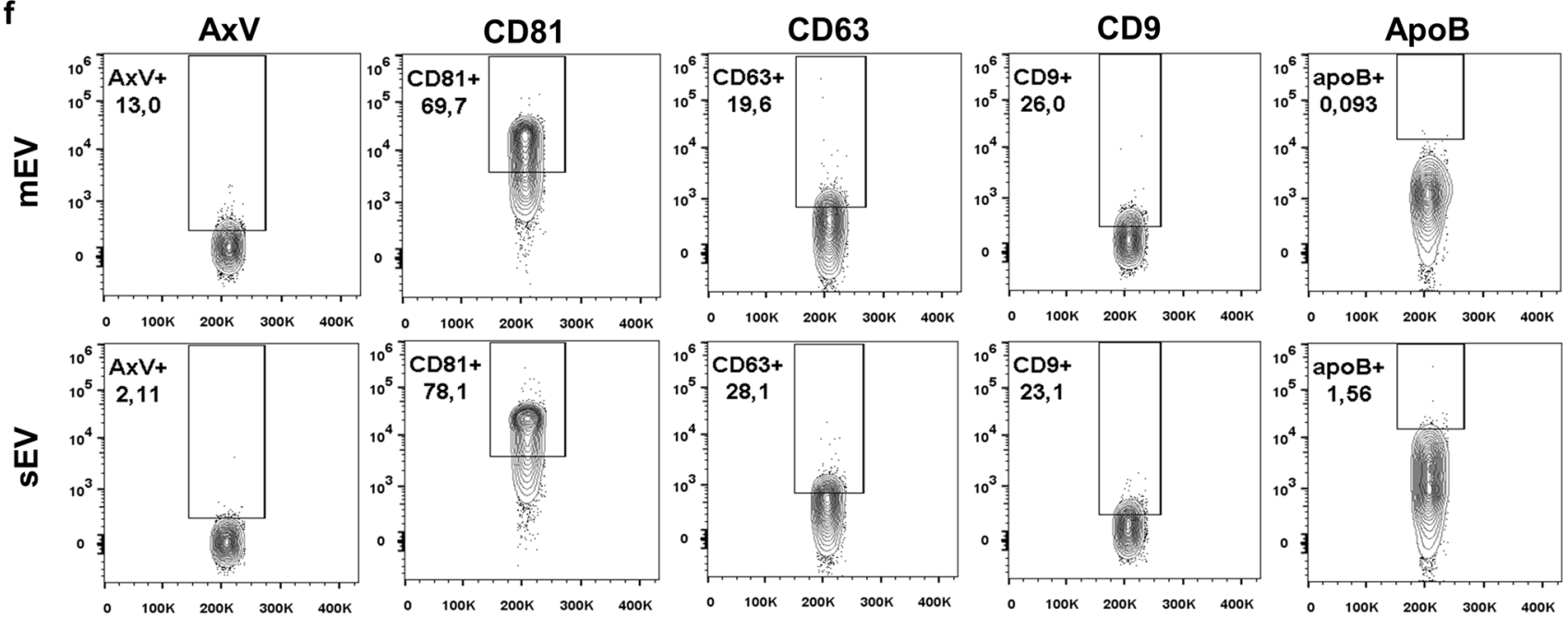

g

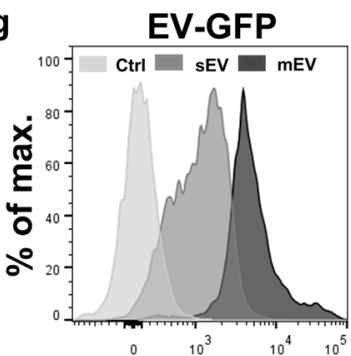

h
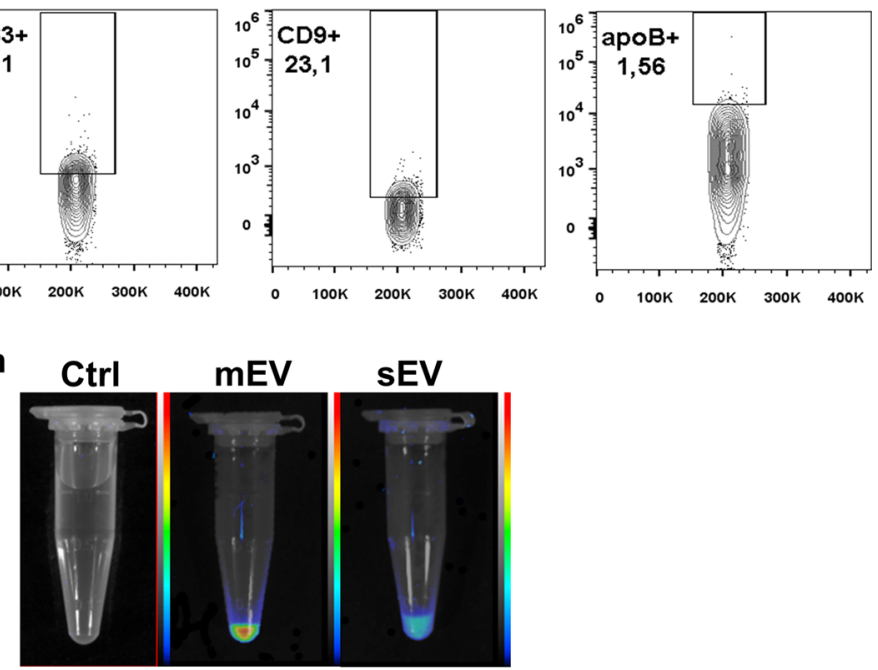

Fig. 4 Characterization of HEK293T-palmGFP-derived EVs. The morphology of EVs was examined by TEM (a and b), the size distribution was determined by NTA (c). Size-based EV fractions were characterized by their particle concentration $(\mathbf{d}, n=3)$ protein/lipid ratios $(\mathbf{e}, n=8)$. The presence of HEK293T-palmGFP-derived EVassociated protein markers and the intrinsic fluorescence of EVs were analyzed by flow cytometry. Identical amounts ( $1 \mu \mathrm{g}$ lipid containing) $\mathrm{mEVs}$ and sEVs were adsorbed onto the surface of latex beads prior to immunostaining (f and $\mathbf{g}$ ). The intrinsic fluorescence of EVs was confirmed by FOBI (h) (intensity values min. 20 to max. 130). mEVs (black symbols), sEVs (grey symbols) 

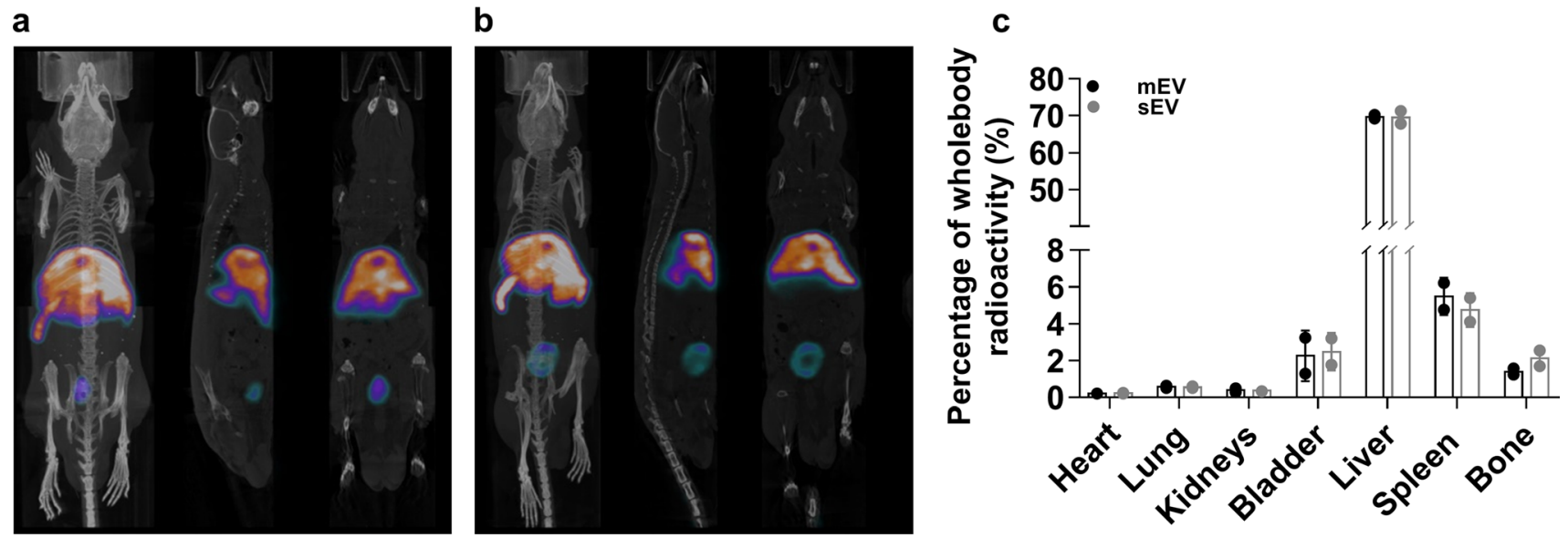

Fig. 5 In vivo SPECT/CT images of the distribution of ${ }^{99 \mathrm{~m}} \mathrm{Tc}$ HYNIC-Duramycin-labeled HEK293T-palmGFP sEV (a) and mEVs (b). The 3D reconstructed and co-registered SPECT and CT images are shown together with sagittal and coronal images (from left to right) (a and b). Quantitative distribution of $\mathrm{sEV}$ and $\mathrm{mEV}$ samples (c). Activities are measured in the specified organs as a percentage of the wholebody radioactivity

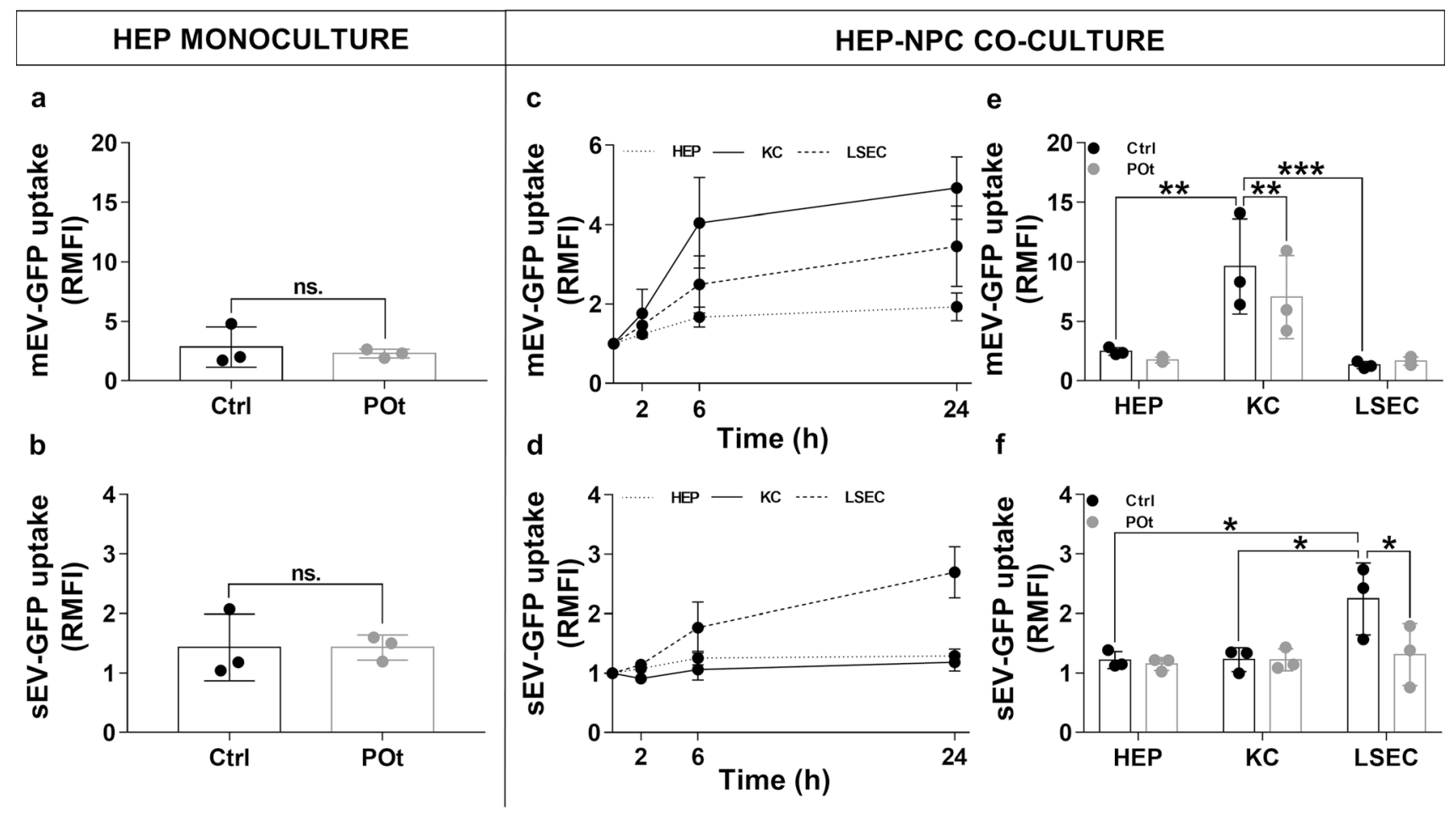

Fig. 6 In vitro uptake of HEK293T-palmGFP-derived EVs by liver cells. The uptake of EVs was examined in HEP monoculture under control and POt conditions. HEPs were able to take up both mEVs (a) and sEVs (b) and the hyperlipidemic condition did not significantly affect the uptake of EVs. Based on our time course experiments (c and $\mathbf{d}$ ), we tested the effect of hyperlipidemic conditions on EV uptake at $24 \mathrm{~h}$ in HEP-NPC co-culture. The mEV uptake of KCs (e) and SEV of LSECs (f) decreased significantly as a result of the FFA treatment. Values are reported as mean \pm SD. $p$ values were calculated either by paired $T$ test (a and $\mathbf{b}$ ) or by two-way ANOVA and Tukey's multiple comparisons post-hoc test (e and f). $n_{\mathrm{Ctrl}}=3$, $n_{\mathrm{POt}}=3, n s$ not-significant, $* p<0.05, * * p<0.01, * * * p<0.001$ 


\section{Discussion}

The goal of the present study was to examine the dynamics of EV release and uptake by liver cells. Importantly, here, we report for the first time the cell type-specific involvement of liver cells (including HEPs and the non-parenchymal KCs and LSECs) in the release and uptake of mEVs and sEVs. While mEVs were primarily taken up by KCs, sEV uptake was the highest in LSECs. Both $\mathrm{mEV}$ and sEV internalization was reduced under hyperlipidemic conditions.

The liver is considered to be a natural scavenger of EVs, independently of the species of origin of EVs. Wiklander and colleagues studied the biodistribution of EVs of different species origins [39]. They found that EVs from human (HEK293T) and rat (OLN-93) cell lines showed the same biodistribution pattern as compared to EVs from a mouse C1C12 cell line. Usually, the role of phagocytic innate immune cells is emphasized in EV elimination. Surprisingly, however, even after clodronate liposome-mediated depletion of macrophages, B16BL6-derived EVs still accumulated in the liver [12]. This suggests that cells other than macrophages contribute to EV elimination. Our in vitro experiments with HEP-NPC co-culture provide evidence that besides liver resident macrophages (KC), HEPs and LSECs are also able to take up EVs. KCs are known to have a key role in eliminating insoluble particles $(\geq 100 \mathrm{~nm}$ ) by phagocytosis, while LSECs are known to remove smaller particles $(\leq 100 \mathrm{~nm})$ from the circulation by pinocytosis [14]. In line with this, here, we found that presumably due to their size, mEVs were eliminated more efficiently by KCs and sEVs by LSECs.

Hyperlipidemia is a collective term for elevated levels of TGs, cholesterol, cholesterol esters and plasma lipoproteins [15]. Uptake of increased amounts of dietary fatty acids in the HFD lead to the absorption of enhanced amounts of FFAs from the lumen of the small intestine. Some FFAs are later liberated from the chylomicrons and are carried to tissues in an albumin-bound form. Chylomicrons later undergo numerous additional changes in their composition, and the so-called "chylomicron remnants" are eliminated from the circulation by the liver [41]. HFD is also known to induce insulin resistance [42], which in turn, also results in an increased level of FFAs.

In this work, we induced chronic hyperlipidemia by sustained exposure of mice to HFD (20-30 weeks), and the hyperlipidemic status was confirmed on the basis of elevated levels of both HDL-C and LDL-C/VLDL-C in the circulation of mice. In vitro, to mimic the hyperlipidemic conditions, we applied the commonly used POt model [43, 44]. Importantly, the same FFAs were predominant in both the HFD and in the FFA-containing tissue culture medium. The effect of diet-induced hyperlipidemia on plasma and HEP-derived EVs have been investigated previously [17,
$18,45-48]$. In line with the previous findings, we detected elevated levels of circulating EV in mice kept on a chronic HFD. However, previous works examined mice after a shorter diet period, and they primarily focused on sEVs. Importantly, previous works only used differential centrifugation for EV separation [17, 18, 48]. Our data presented here demonstrate that secondary (non-genetic) hyperlipidemia is linked to both an increased circulating EV number and an elevated hepatic EV release. In addition to EVs, co-isolation of $\mathrm{ApoB}^{+}$particles was also examined. Of the lipoproteins, chylomicrons, LDL, and VLDL particles carry ApoB on their surface. Plasma contains orders of magnitude more lipoproteins $\left(10^{16}\right)$ than EVs $\left(10^{9}\right)$, and HEPs secrete only VLDL particles $[41,49]$. Although ApoB was indeed present in our samples, we would like to emphasize that we collected fasting plasma, therefore its chylomicron concentration was expected to be low according to our recently published data [33]. Moreover, in HEP cell cultures, one has to calculate only with VLDL and not with LDL or chylomicron. Besides potential co-isolation of LDL and VLDL particles, the ApoB positivity in our preparations could have resulted from the formation LDL and/ or VLDL aggregates or EV-lipoprotein complexes (as we have shown earlier [33]). Most importantly, very recently, we have demonstrated the presence of a universal protein corona around EVs, viruses and synthetic lipid nanoparticles in blood plasma which also includes ApoB (in addition to ApoA1, ApoC3 and ApoE and other proteins) [50] suggesting that $\mathrm{ApoB}$ is not a co-isolated contamination but rather an organic part of the natural protein corona of EVs.

Here, we also report that liver cells under hyperlipidemic conditions had impaired uptake functions. Asanuma and colleagues showed with SPIO-MRI technology that rats and patients with NAFLD (a disease caused by hyperlipidemia) have reduced KC phagocytic function [19]. However, until our work, there has been no data on the possible interference of sEV uptake $(\sim 100 \mathrm{~nm}$ particles) with hyperlipidemia. Limitations of this study is the reported difference between the HDL-LDL balance in mice and in humans (mice lack of the cholesteryl ester transfer protein, an enzyme that transports cholesterol from HDL to LDL, [41]). However, in spite of the above limitations, our data highlight the potential association of EVs and hyperlipidemia.

While the EV field is expanding very rapidly and EVs are considered as very promising potential therapeutic vehicles and agents, rapid elimination of EVs from the circulation is a major limitation of the therapeutic application of EVs. The only exception being the category of liver diseases in the cases of which the natural hepatic docking of EVs eliminates the necessity of specific EV targeting. As a solution, local administration of EVs has been suggested in different studies [51-53]. A deeper understanding of the EV scavenger processes by individual liver cell 
populations may enable the development of therapeutic approaches with blocking or targeting different liver cell populations. Given that hyperlipidemia represents a major health problem in Western countries where reduced daily exercise and Westernized food leads to elevated levels of lipoproteins and FFAs in the circulation, the effect of hyperlipidemia on circulating EV levels may also have multiple implications. In studies on circulating EVs, not only the momentary dietary status (fasting versus postprandial) should be taken into account as we demonstrated previously [33], but also the fat content of the diet (e.g. that of a ketogenic diet) should be taken into account when considering baseline reference EV levels. In addition, elevated levels of circulating EVs under sustained hyperlipidemic conditions may have strong implication in different processes (e.g. in blood coagulation).

The ability to track and measure EVs biodistribution in vivo is essential for utilizing EVs as potential therapeutic vehicles. However, noninvasive methods have been lacking to determine biodistribution of EVs. Optical imaging for tracking EVs is hindered by limited tissue penetration of fluorescent and bioluminescent signals, as well as impractical quantitative analysis. Attempts to radiolabel EVs for SPECT and PET imaging are still in their infancy [7, 8, 54-57]. Duramycin is a phosphatidylethanolamine (PE)-binding peptide which was recently suggested for the radiolabeling of EVs (Clodagh O'Neill, Zhonglin Liu, Róisín Dwyer, Virtual ISEV workshop EV imaging in vivo September 10-11th, 2020). The rationale behind using duramycin for labeling of EVs is that it has high affinity and specificity to bind phosphatidylethanolamine (PE), an abundant phospholipid in EVs [30]. It has been believed that externalization of a significant portion of $\mathrm{PE}$ and phosphatidylserine (PS) from the inner to the outer leaflet of membrane is a key feature of EV formation. As a 19-amino-acid tetracyclic peptide, duramycin is featured with low molecular weight, highly stable structure, high binding affinity to the head group of PE, and higher binding percentage of EVs than cells. Our preliminary labeling results in this study and previous data acquired by Liu and his associates demonstrate that ${ }^{99 \mathrm{~m}} \mathrm{Tc}$-labeled EVs with high yield and stability can be achieved via the specific duramycin-PE binding approach. Of note, the observed distribution in this study highly resembles that of ${ }^{99 \mathrm{~m}} \mathrm{Tc}$ tricarbonyl-labeled erythrocyte-derived EVs [58]. Unlike ${ }^{99 \mathrm{~m}} \mathrm{Tc}$-Duramycin binding to phospholipids of EV membrane, ${ }^{99 \mathrm{~m}} \mathrm{Tc}$-tricarbonyl binds to several amino acids in membrane proteins, which may change the targeting features and biodistributions of EVs. Thus, SPECT imaging with ${ }^{99 \mathrm{~m}} \mathrm{Tc}$-Duramycin-EVs may provide an approach with excellent sensitivity, easier quantification, and less impact on EV biological characteristics in EVs research and clinical translation.
In conclusion, our results showing differential involvement of liver cell types in EV uptake and release, may contribute to the development of improved EV-based drug delivery systems. ${ }^{99 \mathrm{~m}} \mathrm{Tc}$-Duramycin-EVs holds promise as a unique method to track and measure EV homing and biodistribution systematically and noninvasively.

Supplementary Information The online version contains supplementary material available at https://doi.org/10.1007/s00018-021-03969-6.

Acknowledgements We thank Ildiko Horváth for her help with in vivo biodistribution and FOBI measurements.

Author contributions Conceptualization: EIB, KN, VT; methodology: $\mathrm{KN}, \mathrm{VT}, \mathrm{TV}, \mathrm{ÁK}, \mathrm{ZV}, \mathrm{NH}, \mathrm{CPO}, \mathrm{RMD}, \mathrm{ZL}$; formal analysis and investigation: KN, VT, TV, ÁK, ZV, NH, DL, AK; writing-original draft preparation: KN, EIB, VT; writing — review and editing: all authors; funding acquisition: EIB, VT, ZV; resources: EIB, ZV, DM, KSz, PL.

Funding Open access funding provided by Semmelweis University. This work was supported by National Research, Development and Innovation Office NKFIH, Hungary, NVKP_16-1-2016-0017, ÚNKP-19-4-SE-09 and János Bolyai Research Scholarship, the Hungarian Scientific Research Fund (OTKA K120237), VEKOP2.3.2-16-2016-00002, VEKOP-2.3.3-15-2017-00016, H2020-MSCAITN-2017-722148 TRAIN EV and Higher Education Excellence Program (FIKP) Therapeutic Thematic Programme. This project has received funding from the European Union's Horizon 2020 Research and Innovation Programme under grant agreement No 739593. Zoltán Varga was supported by the János Bolyai Research Fellowship, and the ÚNKP-20-5 Bolyai + New National Excellence Program of the Ministry for Innovation and Technology from the source of the National Research, Development and Innovation Fund. Péter Lórincz was supported by Hungarian Academy of Sciences (PPD-222/2018). Radiolabeling study of EVs with ${ }^{99 \mathrm{~m}}$ Tc performed at the University of Arizona was supported by Harrison and CATHERINE Barrett Cancer Imaging Grant, and NIH/NIBIB P41EB002035 grant.

Availability of data and materials All data generated or analyzed during this study are included in this published article and its supplementary information file. We have submitted all relevant data of our experiments to the EV-TRACK knowledgebase (EV-TRACK ID: EV210178).

Code availability Not applicable.

\section{Declarations}

Conflict of interest EIB is member of the Advisory Board of Sphere Gene Therapeutics Inc. (Boston, MA, US).

Ethics approval All animal experiments followed the European Union's Council Directive (86/609/EEC) and were approved by the Semmelweis University's Institutional Animal Care and Use Commitee (PE/ EA/1655-7/2018).

Consent to participate Not applicable.

Consent for publication Not applicable.

Open Access This article is licensed under a Creative Commons Attribution 4.0 International License, which permits use, sharing, 
adaptation, distribution and reproduction in any medium or format, as long as you give appropriate credit to the original author(s) and the source, provide a link to the Creative Commons licence, and indicate if changes were made. The images or other third party material in this article are included in the article's Creative Commons licence, unless indicated otherwise in a credit line to the material. If material is not included in the article's Creative Commons licence and your intended use is not permitted by statutory regulation or exceeds the permitted use, you will need to obtain permission directly from the copyright holder. To view a copy of this licence, visit http://creativecommons. org/licenses/by/4.0/.

\section{References}

1. György B, Szabó TG, Pásztói M et al (2011) Membrane vesicles, current state-of-the-art: emerging role of extracellular vesicles. Cell Mol Life Sci 68:2667-2688. https://doi.org/10.1007/ s00018-011-0689-3

2. Wolf $P$ (1967) The nature and significance of platelet products in human plasma. Br J Haematol 13:269-288. https://doi.org/10. 1111/j.1365-2141.1967.tb08741.x

3. Raposo G, Nijman HW, Stoorvogel W et al (1996) B lymphocytes secrete antigen-presenting vesicles. J Exp Med 183:1161-1172. https://doi.org/10.1084/jem.183.3.1161

4. Wolfers J, Lozier A, Raposo G et al (2001) Tumor-derived exosomes are a source of shared tumor rejection antigens for CTL cross-priming. Nat Med 7:297-303. https://doi.org/10.1038/85438

5. Malloci M, Perdomo L, Veerasamy M et al (2019) Extracellular vesicles: mechanisms in human health and disease. Antioxidants Redox Signal 30:813-856. https://doi.org/10.1089/ars.2017.7265

6. Théry C, Witwer KW, Aikawa E et al (2018) Minimal information for studies of extracellular vesicles 2018 (MISEV2018): a position statement of the International Society for Extracellular Vesicles and update of the MISEV2014 guidelines. J Extracell Vesicles. https://doi.org/10.1080/20013078.2018.1535750

7. Varga Z, Gyurkó I, Pálóczi K et al (2016) Radiolabeling of extracellular vesicles with $99 \mathrm{mtc}$ for quantitative in vivo imaging studies. Cancer Biother Radiopharm 31:168-173. https://doi.org/10. 1089/cbr.2016.2009

8. Hwang DW, Choi H, Jang SC et al (2015) Noninvasive imaging of radiolabeled exosome-mimetic nanovesicle using $99 \mathrm{mTc}$ HMPAO. Sci Rep. https://doi.org/10.1038/srep15636

9. Morishita M, Takahashi Y, Nishikawa M et al (2015) Quantitative analysis of tissue distribution of the B16BL6-derived exosomes using a streptavidin-lactadherin fusion protein and iodine-125-labeled biotin derivative after intravenous injection in mice. J Pharm Sci. https://doi.org/10.1002/jps.24251

10. Smyth T, Kullberg M, Malik N et al (2015) Biodistribution and delivery efficiency of unmodified tumor-derived exosomes. J Control Release. https://doi.org/10.1016/j.jconrel.2014.12.013

11. Takahashi Y, Nishikawa M, Shinotsuka H et al (2013) Visualization and in vivo tracking of the exosomes of murine melanoma B16-BL6 cells in mice after intravenous injection. J Biotechnol. https://doi.org/10.1016/j.jbiotec.2013.03.013

12. Imai T, Takahashi Y, Nishikawa M et al (2015) Macrophagedependent clearance of systemically administered B16BL6derived exosomes from the blood circulation in mice. J Extracell Vesicles 4:1-8. https://doi.org/10.3402/jev.v4.26238

13. Lai CP, Mardini O, Ericsson M et al (2014) Dynamic biodistribution of extracellular vesicles in vivo using a multimodal imaging reporter. ACS Nano 8:483-494. https://doi.org/10.1021/nn404945r

14. Zhang YN, Poon W, Tavares AJ et al (2016) Nanoparticle-liver interactions: cellular uptake and hepatobiliary elimination. J
Control Release 240:332-348. https://doi.org/10.1016/j.jconrel. 2016.01.020

15. Hill MF, Bordoni B (2020) Hyperlipidemia. StatPearls Publishing. https://www.ncbi.nlm.nih.gov/books/NBK559182/. Accessed 07 Feb 2021

16. Mendis S, Puska P, Norrving B (2011) Global atlas on cardiovascular disease prevention and control: policies, strategies and interventions. World Health Organization, Geneva

17. Kakazu E, Mauer AS, Yin M, Malhi H (2016) Hepatocytes release ceramide-enriched pro-inflammatory extracellular vesicles in an IRE1 $\alpha$-dependent manner. J Lipid Res 57:233-245. https://doi. org/10.1194/jlr.M063412

18. Povero D, Eguchi A, Li H et al (2014) Circulating extracellular vesicles with specific proteome and liver microRNAs are potential biomarkers for liver injury in experimental fatty liver disease. PLoS ONE. https://doi.org/10.1371/journal.pone.0113651

19. Asanuma T, Ono M, Kubota K et al (2010) Super paramagnetic iron oxide MRI shows defective Kupffer cell uptake function in non-alcoholic fatty liver disease. Gut 59:258-266. https://doi.org/ 10.1136/gut.2009.176651

20. György B, Pálóczi K, Kovács A et al (2014) Improved circulating microparticle analysis in acid-citrate dextrose (ACD) anticoagulant tube. Thromb Res 133:285-292. https://doi.org/10.1016/j. thromres.2013.11.010

21. Spitler KM, Shetty SK, Cushing EM et al (2021) Regulation of plasma triglyceride partitioning by adipose-derived ANGPTL4 in mice. Sci Rep 11:1-15. https://doi.org/10.1038/ s41598-021-87020-5

22. Cabral F, Miller CM, Kudrna KM et al (2018) Purification of hepatocytes and sinusoidal endothelial cells from mouse liver perfusion. J Vis Exp. https://doi.org/10.3791/56993

23. Hoh CH, Tan YH, Gana YH (2019) Protective role of Kupffer cells and macrophages in Klebsiella pneumoniae-induced liver abscess disease. Infect Immun. https://doi.org/10.1128/IAI. 00369-19

24. Keemink J, Oorts M, Annaert P (2014) Primary hepatocytes in sandwich culture. Methods Mol Biol 1250:175-188. https://doi. org/10.1007/978-1-4939-2074-7_12

25. Théry C, Amigorena S, Raposo G, Clayton A (2006) Isolation and characterization of exosomes from cell culture supernatants and biological fluids. Curr Protoc Cell Biol. https://doi.org/10.1002/ 0471143030.cb0322s30

26. György B, Szabó TG, Turiák L et al (2012) Improved flow cytometric assessment reveals distinct microvesicle (cell-derived microparticle) signatures in joint diseases. PLoS ONE. https:// doi.org/10.1371/journal.pone.0049726

27. Visnovitz T, Osteikoetxea X, Sódar BW et al (2019) An improved 96 well plate format lipid quantification assay for standardisation of experiments with extracellular vesicles. J Extracell Vesicles 8:1565263. https://doi.org/10.1080/20013078.2019.1565263

28. Suárez H, Gámez-Valero A, Reyes R et al (2017) A beadassisted flow cytometry method for the semi-quantitative analysis of extracellular vesicles. Sci Rep. https://doi.org/10.1038/ S41598-017-11249-2

29. Lynch RW, Hawley CA, Pellicoro A et al (2018) An efficient method to isolate Kupffer cells eliminating endothelial cell contamination and selective bias. J Leukoc Biol 104:579-586. https:// doi.org/10.1002/JLB.1TA0517-169R

30. Zhao M, Li Z, Bugenhagen S (2008) 99mTc-labeled duramycin as a novel phosphatidylethanolamine- binding molecular probe. J Nucl Med 49:1345-1352. https://doi.org/10.2967/jnumed.107. 048603

31. Kitka D, Mihály J, Fraikin JL et al (2019) Detection and phenotyping of extracellular vesicles by size exclusion chromatography coupled with on-line fluorescence detection. Sci Rep 9:1-7. https://doi.org/10.1038/s41598-019-56375-1 
32. Gallou-Kabani C, Vigé A, Gross MS et al (2007) C57BL/6J and $\mathrm{A} / \mathrm{J}$ mice fed a high-fat diet delineate components of metabolic syndrome. Obesity 15:1996-2005. https://doi.org/10.1038/oby. 2007.238

33. Sódar BW, Kittel Á, Pálóczi K et al (2016) Low-density lipoprotein mimics blood plasma-derived exosomes and microvesicles during isolation and detection. Sci Rep. https://doi.org/10.1038/ srep24316

34. Suades R, Padró T, Vilahur G, Badimon L (2012) Circulating and platelet-derived microparticles in human blood enhance thrombosis on atherosclerotic plaques. Thromb Haemost 108:1208-1219. https://doi.org/10.1160/TH12-07-0486

35. Tan M, Yan HB, Li JN et al (2016) Thrombin stimulated plateletderived exosomes inhibit platelet-derived growth factor receptorbeta expression in vascular smooth muscle cells. Cell Physiol Biochem 38:2348-2365. https://doi.org/10.1159/000445588

36. Montgomery Bissell D, Hammaker LE, Meyer UA (1973) Parenchymal cells from adult rat liver in nonproliferating monolayer culture. J Cell Biol. https://doi.org/10.1083/jcb.59.3.722

37. Rowell DL, Eckmann L, Dwinell MB et al (1997) Human hepatocytes express an array of proinflammatory cytokines after agonist stimulation or bacterial invasion. Am J Physiol Liver Physiol. https://doi.org/10.1152/ajpgi.1997.273.2.G322

38. Li H, Zhou Y, Wang $\mathrm{H}$ et al (2020) Crosstalk between liver macrophages and surrounding cells in nonalcoholic steatohepatitis. Front Immunol 11:1169. https://doi.org/10.3389/fimmu.2020. 01169

39. Wiklander OPB, Nordin JZ, O'Loughlin A et al (2015) Extracellular vesicle in vivo biodistribution is determined by cell source, route of administration and targeting. J Extracell Vesicles 4:1-13. https://doi.org/10.3402/jev.v4.26316

40. Kmieć Z (2001) Cooperation of liver cells in health and disease. Springer, Berlin

41. Feingold KR, Grunfeld C (2000) Introduction to Lipids and Lipoproteins. MDText.com, Inc. https://www.ncbi.nlm.nih.gov/books/ NBK305896/. Accessed 19 Jan 2021

42. Surwit RS, Kuhn CM, Cochrane C et al (1988) Diet-induced type II diabetes in C57BL/6J mice. Diabetes. https://doi.org/10.2337/ diab.37.9.1163

43. Moravcová A, Červinková Z, Kučera O et al (2015) The effect of oleic and palmitic acid on induction of steatosis and cytotoxicity on rat hepatocytes in primary culture. Physiol Res. https://doi.org/ 10.33549/physiolres.933224

44. Ricchi M, Odoardi MR, Carulli L et al (2009) Differential effect of oleic and palmitic acid on lipid accumulation and apoptosis in cultured hepatocytes. J Gastroenterol Hepatol. https://doi.org/10. 1111/j.1440-1746.2008.05733.x

45. Pirro M, Schillaci G, Paltriccia $R$ et al (2006) Increased ratio of CD31+/CD42- microparticles to endothelial progenitors as a novel marker of atherosclerosis in hypercholesterolemia. Arterioscler Thromb Vasc Biol 26:2530-2535. https://doi.org/10.1161/ 01.ATV.0000243941.72375.15
46. Nomura S, Suzuki M, Katsura K et al (1995) Platelet-derived microparticles may influence the development of atherosclerosis in diabetes mellitus. Atherosclerosis 116:235-240. https://doi.org/ 10.1016/0021-9150(95)05551-7

47. Li J, Liu H, Mauer AS et al (2019) Characterization of cellular sources and circulating levels of extracellular vesicles in a dietary murine model of nonalcoholic steatohepatitis. Hepatol Commun 3:1235-1249. https://doi.org/10.1002/hep4.1404

48. Povero D, Eguchi A, Niesman IR et al (2013) Lipid-induced toxicity stimulates hepatocytes to release angiogenic microparticles that require vanin-1 for uptake by endothelial cells. Sci Signal. https://doi.org/10.1126/scisignal.2004512

49. Simonsen JB (2017) What are we looking at? Extracellular vesicles, lipoproteins, or both? Circ Res 121:920-922. https://doi.org/ 10.1161/CIRCRESAHA.110.226258

50. Tóth EÁ, Turiák L, Visnovitz T et al (2021) Formation of a protein corona on extracellular vesicles in blood plasma. J Extracell Vesicles. https://doi.org/10.1002/jev2.12140

51. Berger A, Araújo-Filho I, Piffoux M et al (2021) Local administration of stem cell-derived extracellular vesicles in a thermoresponsive hydrogel promotes a pro-healing effect in a rat model of colo-cutaneous post-surgical fistula. Nanoscale. https://doi.org/ 10.1039/D0NR07349K

52. Zhang S, Chu WC, Lai RC et al (2016) Exosomes derived from human embryonic mesenchymal stem cells promote osteochondral regeneration. Osteoarthr Cartil 24:2135-2140. https://doi.org/10. 1016/j.joca.2016.06.022

53. Han C, Zhou J, Liang C et al (2019) Human umbilical cord mesenchymal stem cell derived exosomes encapsulated in functional peptide hydrogels promote cardiac repair. Biomater Sci 7:2920 2933. https://doi.org/10.1039/c9bm00101h

54. Gangadaran P, Hong CM, Oh JM et al (2018) In vivo non-invasive imaging of radio-labeled exosome-mimetics derived from red blood cells in mice. Front Pharmacol. https://doi.org/10.3389/ fphar.2018.00817

55. Molavipordanjani S, Khodashenas S, Abedi SM et al (2020) 99mTc-radiolabeled HER2 targeted exosome for tumor imaging. Eur J Pharm Sci. https://doi.org/10.1016/j.ejps.2020.105312

56. Rashid MH, Borin TF, Ara R et al (2020) Generation of novel diagnostic and therapeutic exosomes to detect and deplete protumorigenic M2 macrophages. Adv Ther. https://doi.org/10.1002/ adtp.201900209

57. Takahashi Y, Nishikawa M, Takakura Y (2016) Analysis and control of in vivo kinetics of exosomes for the development of exosome-based DDS. Yakugaku Zasshi 136:49

58. Larson MC, Woodliff JE, Hillery CA et al (2012) Phosphatidylethanolamine is externalized at the surface of microparticles. Biochim Biophys Acta Mol Cell Biol Lipids 1821. https://doi.org/10. 1016/j.bbalip.2012.08.017

Publisher's Note Springer Nature remains neutral with regard to jurisdictional claims in published maps and institutional affiliations. 